\title{
The Stokesian hydrodynamics of flexing, stretching filaments
}

\author{
Michael J. Shelley ${ }^{a}$, Tetsuji Ueda ${ }^{b, *}$ \\ ${ }^{a}$ Courant Institute of Mathematical Sciences, New York University, New York, NY 10012, USA \\ ${ }^{\mathrm{b}}$ Department of Mathematics and Computer Science, Kent State University, Kent, OH 44242, USA
}

Received 3 May 1999; received in revised form 10 April 2000; accepted 2 June 2000

Communicated by C.K.R.T. Jones

\begin{abstract}
A central element of many fundamental problems in physics and biology lies in the interaction of a viscous fluid with slender, elastic filaments. Examples arise in the dynamics of biological fibers, the motility of microscopic organisms, and in phase transitions of liquid crystals. When considering the dynamics on the scale of a single filament, the surrounding fluid can often be assumed to be inertialess and hence governed by the Stokes' equations. A typical simplification then is to assume a local relation, along the filament, between the force per unit length exerted by the filament upon the fluid and the velocity of the filament. While this assumption can be justified through slender-body theory as the leading-order effect, this approximation is only logarithmically separated (in aspect ratio) from the next-order contribution capturing the first effects of non-local interactions mediated by the surrounding fluid; non-local interactions become increasingly important as a filament comes within proximity to itself, or another filament. Motivated by a pattern forming system in isotropic to smectic-A phase transitions, we consider the non-local Stokesian dynamics of a growing elastica immersed in a fluid. The non-local interactions of the filament with itself are included using a modification of the slender-body theory of Keller and Rubinow. This modification is asymptotically equivalent, and removes an instability of their formulation at small, unphysical length-scales. Within this system, the filament lives on a marginal stability boundary, driven by a continual process of growth and buckling. Repeated bucklings result in filament flex, which, coupled to the non-local interactions and mediated by elastic response, leads to the development of space-filling patterns. We develop numerical methods to solve this system accurately and efficiently, even in the presence of temporal stiffness and the close self-approach of the filament. While we have ignored many of the thermodynamic aspects of this system, our simulations show good qualitative agreement with experimental observations. Our results also suggest that non-locality, induced by the surrounding fluid, will be important to understanding the dynamics of related filament systems. (C) 2000 Elsevier Science B.V. All rights reserved.
\end{abstract}

Keywords: Stokesian fluid; Smectic-A phase transition; Slender-body theory

\section{Introduction}

Many problems in physics and biology are characterized by the dynamics of slender, elastic filaments (elastica) immersed in a Stokesian fluid (a flow of negligible fluid inertia). Examples of such systems are smectic-A $\left(\mathrm{S}_{\mathrm{A}}\right)$ liquid crystal (LC) filaments grown from an isotropic phase [22,23], self-assemblage of phospho-lipid bilayer tubes

\footnotetext{
* Corresponding author. Fax: +1-330-672-7824

E-mail address: ueda@mcs.kent.edu (T. Ueda).
} 


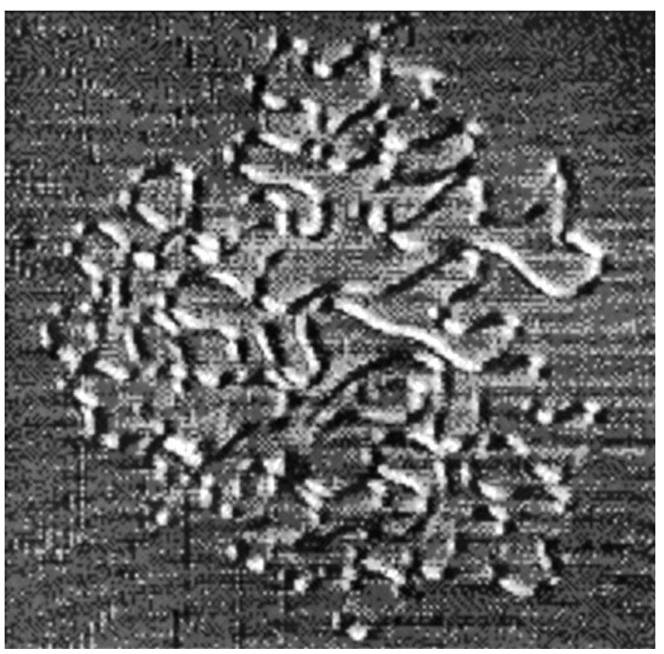

Fig. 1. The growing $\mathrm{S}_{\mathrm{A}}$ filament in an isotropic fluid. Courtesy of P. Palffy-Muhoray (LCI, Kent State University).

[25], folding dynamics of inextensible stiff polymers [10], motility of microscopic organisms driven by undulating flagella $[4,20]$, and twisting and writhing of bacterial macrofibers [11,21].

As a beautiful example of what can result from the interaction of a slender, flexible object with a surrounding Stokesian fluid, consider Fig. 1. It shows a snapshot of a growing $S_{A}$ filament from the experiments of Palffy-Muhoray et al. [23]. (The dynamics of self-assembling bilayer tubes studied by Rudolph et al. [25] show a somewhat similar pattern formation.) It has achieved this complicated and space-filling patterning through a continuous process of filament growth and buckling. The repeated bucklings are most pronounced on the pattern periphery, leaving behind an inner region of "packed", closely spaced sections of filament with slower transverse motion. The underlying mechanisms driving this dynamics seem partly thermodynamical and partly fluid mechanical.

As demonstrated by Palffy-Muhoray et al., the undercooling of certain LC materials can drive an isotropic to smectic-A ( $\left(-S_{A}\right)$ phase transition, with the surrounding isotropic fluid taken up by an evolving $S_{A}$ filament (the filament itself is a metastable state, and the material sample eventually transitions to a focal conic structure). Volumetric uptake of material causes growth of the filament length $(L \sim 5 \rightarrow 1000 \mu \mathrm{m})$, while its cross-sectional radius remains fixed $(a \sim 1.5 \mu \mathrm{m})$. Experimental measurements [23] indicate

$$
L(t)=L_{\circ} \mathrm{e}^{\sigma t},
$$

where $\sigma$ is the constant exponential growth rate. This growth law is consistent with a uniform mass flux through the filament surface area. The internal structure of the filament consists of concentric layers of $\mathrm{S}_{\mathrm{A}}$ with the LC directors normal to the layers, radially about the centerline axis (see $[6,23])$. With this preferred LC conformation for a straight filament, any bends in the centerline lead to non-radial splay deformations in the $\mathrm{S}_{\mathrm{A}}$ structure. Weak deformations, where the bending arclength curvature $\kappa$ is small relative to the filament radius, $\kappa \ll 1 / a$, would lead to elastic restoring forces. Recently, E and Palffy-Muhoray [7] have posed a solidification model for the I-S phase transition, and developed a detailed picture of the mechanisms underlying selection of the filament radius, permeation current, and other features.

What of fluid mechanics? Fluid drag will impart tensile forces on an initially straight, but growing filament, making it susceptible to buckling. The buckling is limited in its length-scale by the elastic restoring forces of smectic layering. Continued growth leads to flexing, or a continual process of buckling. However, the non-local 

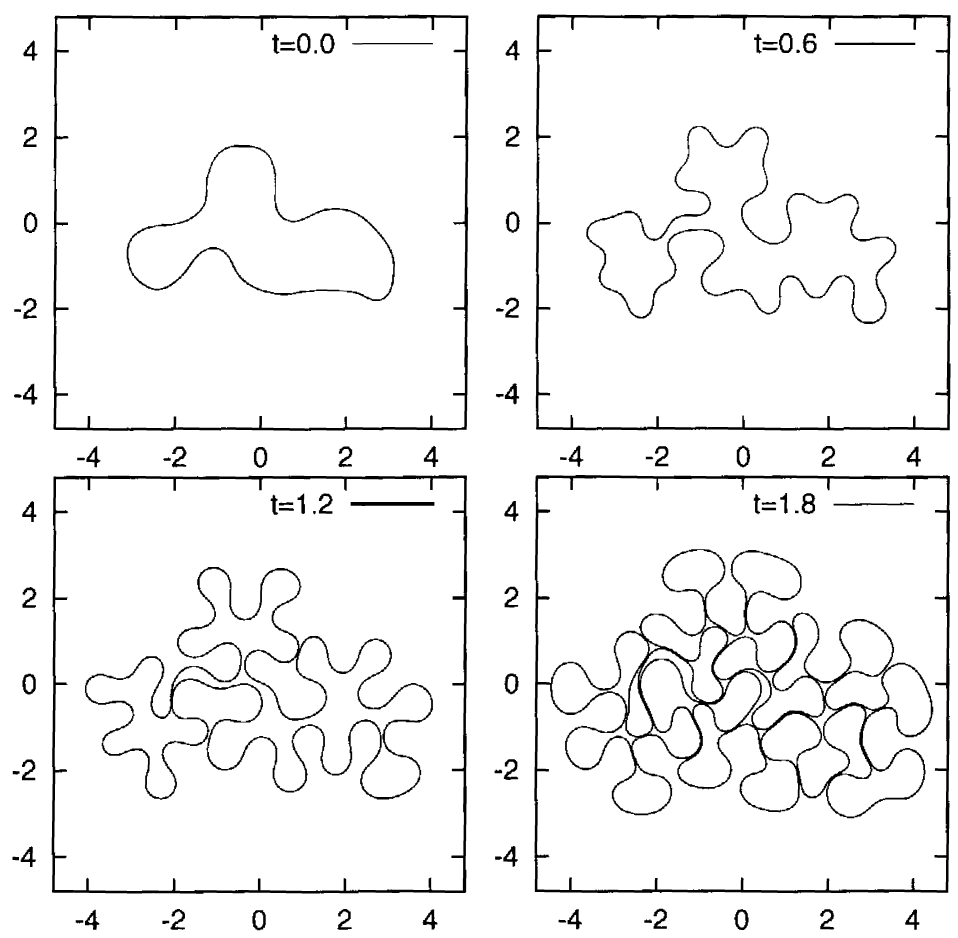

Fig. 2. Numerical simulation of a growing and flexing filament.

interactions of the filament with itself, through the intervening fluid, should strongly mediate the resulting pattern formation. For example, the fluid should slow, or even stop, the filament's transverse motion as two disparate sections approach one another in the center regions of the pattern.

In this paper, we present a novel fluid dynamical model which captures the essence of the evolving pattern. Snapshots of the evolving filament centerline, taken from a sample numerical simulation of our model, are shown in Fig. 2. The computed solution highlights the increasing complexity in the curve shape, very similar in character to that observed in the physical experiments. The curve grows in length, flexes, and is apparently self-avoiding and space-filling. We observe packing of the curve in the interior region of the forming pattern, while folds in the curve on the periphery expand further outwards. Repeated bucklings of the curve on the periphery suggest that the system lives on a marginal stability boundary.

Flow in the isotropic fluid (with Reynolds' number estimated by the experimentalists to be $R e \sim 10^{-4}$ ) is described by the incompressible Stokes' equation:

$$
\nabla p=\mu \Delta \mathbf{u}, \quad \nabla \cdot \mathbf{u}=0,
$$

where $\mu$ is the Newtonian viscosity, $\mathbf{u}$ the fluid velocity field, and $p$ the pressure. Since the flow is viscously dominated, forces on the fluid result in velocity and not acceleration and stipulate a linear relationship between the force and fluid velocity.

Using slender-body theory and a no-slip boundary condition on the fluid-filament interface, the Stokes' equations can be asymptotically reduced to obtain a force-velocity relation of the form (see $[16,19])$

$$
8 \pi \mu \mathbf{V}(s, t)=-c \mathbf{A}(s, t) \cdot \mathbf{f}(s, t)+\mathbf{G}[\mathbf{f}(s, t)],
$$


where $s$ is the arclength, $\mathbf{V}(s, t)$ the translational velocity of the filament, and $\mathbf{f}(s, t)$ the drag force per unit length. The "anisotropy matrix" is given by $\mathbf{A}(s, t)=\mathbf{I}+\hat{\mathbf{s}}(s, t) \hat{\mathbf{s}}(s, t)$, where $\mathbf{I}$ is the identity and $\hat{\mathbf{s}}(s, t)$ is the unit tangent vector along the filament centerline. The linear operator $\mathbf{G}$ involves a modified Stokeslet distribution over the filament length, and captures the non-local influence of fluid drag, where motions of separated segments of the filament are mediated by the surrounding fluid. The parameter $c \sim 2 \log (1 / \epsilon)$ scales $\log$ arithmically with the aspect ratio $\epsilon=a / L$, where $0<\epsilon \ll 1$ in the slender-body limit.

Note that if $\mathbf{f}$ were now specified as a function of the filament conformation, then $\mathbf{V}$ could in principal be calculated and the filament position updated. Note too that the resulting filament dynamics is non-local, nonlinear in filament position, but also closed and self-contained. This is a general feature of Stokes' flows.

In previous work [29], we neglected the non-local term $\mathbf{G}$, resulting in a purely local relationship between $\mathbf{V}$ and $\mathbf{f}$, and specified $\mathbf{f}$ as a sum of tensile and elastic forces. Within this "local drag model", we identified a buckling instability. The tensile and elastic forces in the filament, coupled to local effects of fluid drag, then determine a critical radius of curvature, $r \propto(E / \mu)^{1 / 4}$, where $E$ is the elastic constant. As the radius of curvature of a bend increases with filament growth, and becomes larger than this critical value, the increasing negative tension along the filament induces a buckling instability. This buckling decreases the radius of curvature, the cycle repeats, and results in a flexing curve typified by iterated bucklings at the critical radius.

We note that for the elastica-fluid systems cited earlier, typical analyses also neglect the non-local term G. Indeed, our "local drag model" is very similar to models for polymer dynamics derived from energetics theory in recent works of Goldstein and Langer [10], Goldstein et al. [11] and Bourdieu et al. [2], though these works assumed inextensibility, and used an isotropic $\operatorname{drag}(\mathbf{A}=\mathbf{I})$.

Unfortunately, patterns similar to those observed experimentally by Palffy-Muhoray et al. [23] cannot be obtained from the leading-order, local drag model. Most notable was that its filament centerline was driven to multiple self-intersections. Such self-crossings are, of course, unphysical and reflect the lack of non-local hydrodynamic interactions. In fact, iterated bucklings will persistently bring into close proximity disparate sections of the filament (as it is evident most especially in the inner regions of the pattern), and these interactions will be dominated by non-local hydrodynamics.

Further, as a leading-order theory, the local model suffers also from slow asymptotic convergence, e.g., even for an aspect ratio of size $\epsilon \sim 10^{-4}$, the neglected term has a relative magnitude of $1 / c \sim 0.1$. This brings to question the accuracy of the system where the leading-order non-local term is neglected. The classical formulation for the non-local term $\mathbf{G}$ is the slender-body theory of Keller and Rubinow [19]. Their model is asymptotically accurate to $\mathrm{O}\left(\epsilon^{2} \log \epsilon\right)$ (see [18]) and so does not suffer from slow convergence. We have investigated their model for use in a non-local drag model but have found that coupling their theory to the filament elasticity leads to an ill-posed dynamical system that exhibits unphysical short-length-scale instabilities.

As implied by Fig. 2, we have overcome these difficulties and give here a novel formulation for the leading-order, non-local dynamics of a filament immersed in a Stokesian fluid, and apply it to the special case of the growing $\mathrm{S}_{\mathrm{A}}$ filament. In Section 2, we develop an asymptotic expression for the filament velocity $\mathbf{V}$ in which the unphysical instabilities are eliminated, but which retains the same order of asymptotic accuracy as that of Keller and Rubinow (Eq. (3)). Evolution of the resulting system requires the evaluation of an integral involving a modified Stokeslet distribution along the filament centerline, and the solution of an auxiliary integro-differential equation for the filament tension so as to satisfy a local growth constraint. In Section 3, we study a buckling instability within this model, and from it make a prediction of critical length-scales. We make a comparison of these predictions with simulations of the fully nonlinear model, and expand on differences between the local and non-local descriptions. In Section 4, we study elements of the fully developed patterns found through simulation. In Appendices A-E are contained supporting mathematical materials. This includes establishing the asymptotic consistency of our formulation with that of Keller and Rubinow, the ill-posedness in this dynamical 
setting of the Keller and Rubinow formulation, details of linear stability theory, and a discussion of numerical methods.

\section{The slender-body hydrodynamical model}

Let $\Gamma$ denote the filament centerline. In partial consistency with the experiments, we choose data so that $\Gamma$ lies in a two-dimensional plane, while the fluid motion remains fully three-dimensional (in the experiments, the filament and fluid motion were restricted by two microscope slides separated by narrow spacers).

Let $\mathbf{f}$ be the force per unit length along $\Gamma$, determined from total tensile and elastic forces on the filament. With weak bends, we use Euler-Bernoulli elasticity and obtain the force per unit length [28],

$$
\mathbf{f}=-(T \hat{\mathbf{s}})_{s}+\left(E \kappa_{S} \hat{\mathbf{n}}\right)_{s},
$$

where $T$ is the line tension, $E$ the elastic constant, and $\hat{\mathbf{n}}$ is the unit normal vector along $\Gamma$ (here and elsewhere, subscripts with continuous variables denote partial differentiation). Note that twist elasticity is neglected. For the case considered here, the out-of-plane filament velocity is zero and there are no external fluid flows which would twist the filament. More importantly for the smectic-A filament, while bending would lead to splay deformations, twisting would not deform the LC director field. Twisting should only affect the internal fluid flow in the smectic layering, leading to viscous rather than elastic forces.

Physical underpinnings for the $\mathrm{S}_{\mathrm{A}}$ filament formation are described in [7,23]. In the latter study, a solidification model for the filament growth was developed. With incoming mass flux proportional to its surface area, the filament grows uniformly along its entire length. Uniform growth along the filament leads to a time-dependent arclength,

$$
s(\alpha, t)=\alpha \mathrm{e}^{\sigma t}
$$

where $\sigma$ is the constant exponential growth rate and $\alpha$ is the initial arclength parameter of the filament. Parameter $\alpha$ also serves as a Lagrangian (material) coordinate along $\Gamma$, where we may easily interchange $s$ and $\alpha$ derivatives via $\partial / \partial \alpha=s_{\alpha}(\partial / \partial s)$. Note that Eq. (5) is consistent with the measured exponential growth of $L$, as in Eq. (1).

There is a uniform permeation current through the surface of the filament. This flow has magnitude $v_{t} / S$, where $v=\pi a^{2} L$ is the filament volume and $S=2 \pi a L$ is the filament surface area. The magnitude of this permeation current thus scales as $\mathrm{O}(\epsilon)$ and so is higher-order to our analysis. Thus we neglect it and impose a no-slip boundary condition on the filament surface. The elastic and tensile forces are then exactly balanced by the fluid drag, and the f above may be interpreted as the fluid drag per unit length used in Eq. (3). As a simplification, we assume that the filament forms a closed loop, such that there are no loose ends.

We non-dimensionalize using the following unit quantities: length $r=L_{\circ} /(2 \pi)$ (the radius of a circle with circumference $L_{\circ}$ ), time $t=\sigma^{-1}$ (filament growth rate), and force $F=E / L^{2}$ (filament elasticity). We introduce an effective viscosity,

$$
\bar{\mu}=\frac{\mu \sigma L_{\circ}^{4}}{2 \pi^{3} E},
$$

which represents a ratio between the characteristic fluid drag and the filament elastic force. Arclength is now given by $s(\alpha, t)=\alpha \mathrm{e}^{t}$, the aspect ratio by $\epsilon=a \mathrm{e}^{-t} /(2 \pi)$, where $a$ is the non-dimensional radius, and the logarithmic scaling coefficient by

$$
c=-\log \left(\frac{1}{16} \epsilon^{2} \mathrm{e} \pi^{2}\right) .
$$

Non-dimensional length is given by $L(t)=2 \pi \mathrm{e}^{t}$. Dependent variables are now $2 \pi$-periodic with respect to $\alpha$. 
Let $\mathbf{X}(\alpha, t)$ be coordinates along $\Gamma$. The translational velocity

$$
\mathbf{V}(\alpha, t)=\frac{\mathrm{d}}{\mathrm{d} t} \mathbf{X}(\alpha, t)
$$

describes the kinematics of the filament. Since the filament is immersed in a Stokes' flow, there is a linear relationship between $\mathbf{f}$ and $\mathbf{V}$. Our model uses the following integral relation:

$$
\bar{\mu} \mathbf{V}(\alpha, t)=-\int_{-\pi}^{\pi} \frac{2 s_{\alpha}\left|\sin \left(\alpha^{\prime} / 2\right)\right|}{\left|\mathbf{R}\left(\alpha, \alpha^{\prime}, t\right)\right|} \frac{\left(\mathbf{I}+\hat{\mathbf{R}}\left(\alpha, \alpha^{\prime}, t\right) \hat{\mathbf{R}}\left(\alpha, \alpha^{\prime}, t\right)\right) \cdot \mathbf{f}\left(\alpha+\alpha^{\prime}, t\right)}{\left(4 \sin ^{2}\left(\alpha^{\prime} / 2\right)+y^{2}\right)^{1 / 2}} \mathrm{~d} \alpha^{\prime}-2 \hat{\mathbf{n}}(\alpha, t)(\hat{\mathbf{n}}(\alpha, t) \cdot \mathbf{f}(\alpha, t))
$$

where $y=a \sqrt{\mathrm{e}} / s_{\alpha}$, and $\mathbf{R}\left(\alpha, \alpha^{\prime}, t\right)=\mathbf{X}(\alpha, t)-\mathbf{X}\left(\alpha+\alpha^{\prime}, t\right)$ is the relative vector between two points on $\Gamma$, and $\hat{\mathbf{R}}=\mathbf{R} /|\mathbf{R}|$.

Eq. (9) is the leading-order asymptotic limit for a slender-body immersed in a Stokesian fluid, with an asymptotic accuracy of $\mathrm{O}\left(\epsilon^{2} \log \epsilon\right)$. It is derived from a matched asymptotic expansion using Stokeslets solutions of Chwang and $\mathrm{Wu}$ [5] for the outer flow. Note that the kernel in the integral is periodic with respect to the variable of integration, since the filament here is a closed loop. We rewrite the relation exactly to take the form as given in Eq. (3),

$$
\bar{\mu} \mathbf{V}=-M_{0}(y) \mathbf{A} \cdot \mathbf{f}-2 \hat{\mathbf{n}}(\hat{\mathbf{n}} \cdot \mathbf{f})-\mathbf{K}[\mathbf{f}],
$$

where $M_{0}(y)$ is a zeroth-order toroidal harmonics (see Appendix E) and where

$$
\begin{aligned}
\mathbf{K}[\mathbf{f}]= & \int_{-\pi}^{\pi} \frac{1}{\left(4 \sin ^{2}\left(\alpha^{\prime} / 2\right)+y^{2}\right)^{1 / 2}} \\
& \times\left[\frac{2 s_{\alpha}\left|\sin \left(\alpha^{\prime} / 2\right)\right|}{\left|\mathbf{R}\left(\alpha, \alpha^{\prime}, t\right)\right|}\left(\mathbf{I}+\hat{\mathbf{R}}\left(\alpha, \alpha^{\prime}, t\right) \hat{\mathbf{R}}\left(\alpha, \alpha^{\prime}, t\right)\right) \cdot \mathbf{f}\left(\alpha+\alpha^{\prime}, t\right)-\mathbf{A}(\alpha, t) \cdot \mathbf{f}(\alpha, t)\right] \mathrm{d} \alpha^{\prime}
\end{aligned}
$$

is a linear integral operator on $\mathbf{f}$. Since

$$
M_{0}(y)=2 \log \left(\frac{8}{y}\right)+\mathrm{O}\left(y^{2} \log y\right) \text { for } 0<y \ll 1
$$

(see [14, Eqs. (8.11) and (8.128)]), and comparing with $c$ in Eq. (7), we may equate the logarithmic leading-order terms in Eqs. (3) and (10) by specifying $y=a \sqrt{\mathrm{e}} / s_{\alpha}$.

We show in Appendix A that the Keller and Rubinow formulation [19] is asymptotically equivalent to ours with the same asymptotic accuracy of $\mathrm{O}\left(\epsilon^{2} \log \epsilon\right)$. In fact, their model may be determined by taking the non-singular limit $y \rightarrow 0$ in Eq. (11), which results in a jump discontinuity in the integral kernel. We show in Appendix B that this jump discontinuity leads to higher-wavenumber instabilities. In the high-wavenumber limit, $k \gg 1$, a $k$ th Fourier mode perturbation to the filament centerline curve grows with exponential growth rate $\sigma_{k} \sim+2 k^{4} \log (\epsilon k) / \bar{\mu}$, i.e., modes which scale on the filament radius and shorter are unstable. Such short length-scales are unphysical since they are excluded from the slender-body theory. The presence of this instability, however, leads to an ill-posed model, and numerical simulations with fine discretizations may re-introduce the unstable length-scales back into the system.

By retaining the cut-off scale $y$ in the integral, our model smoothes away the jump discontinuity, while affecting only those modes shorter than the radius $a$ length-scale. We show in Section 3 and Appendix $\mathrm{C}$ that the growth rate is $\sigma_{k} \sim-2 k^{4} / \bar{\mu}$ for higher-wavenumber modes; i.e., the behavior for these short length-scales are dominated by the fourth-order elastic regularization.

In the plane, it is natural to separate the normal and tangential components of the filament motion by taking, as our dynamical variables, the arclength metric $s_{\alpha}$ and tangent angle $\theta$. The $s_{\alpha}-\theta$ pair has been used previously in 
analyzing curve motion (e.g., see $[10,17,27])$, perhaps first by Whitham [30] in analyzing the propagation of shock fronts. From Eq. (8) and the Frenet-Seret relations, we obtain

$$
s_{\alpha t}=s_{\alpha} \hat{\mathbf{s}} \cdot \mathbf{V}_{s},
$$

where time appears as a parameter, since $s_{\alpha t}$ is completely determined by the uniform growth of Eq. (5).

The line tension $T$ is the Lagrange multiplier which constrains the filament motion to satisfy the uniform growth as specified by Eq. (5). Eq. (13) defines a linear integro-differential equation for $T$,

$$
\begin{aligned}
& 2 M_{0}(y) T_{s s}-\left(M_{0}(y)+2\right) \kappa^{2} T+\hat{\mathbf{s}} \cdot \frac{\partial}{\partial s} \mathbf{K}\left[\hat{\mathbf{s}} T_{s}+\hat{\mathbf{n}} \kappa T\right] \\
& =\bar{\mu} \frac{s_{\alpha t}}{s_{\alpha}}-2 M_{0}(y) \kappa_{s}^{2}-\left(3 M_{0}(y)+2\right) \kappa \kappa_{s s}+\hat{\mathbf{s}} \cdot \frac{\partial}{\partial s} \mathbf{K}\left[\hat{\mathbf{n}} \kappa_{s s}-\hat{\mathbf{s}} \kappa \kappa_{s}\right],
\end{aligned}
$$

which must be solved at each time, given the curve shape $\mathbf{X}$.

The tangent angle is defined by

$$
\hat{\mathbf{s}}=\hat{\mathbf{x}} \cos \theta+\hat{\mathbf{y}} \sin \theta, \quad \hat{\mathbf{n}}=-\hat{\mathbf{x}} \sin \theta+\hat{\mathbf{y}} \cos \theta,
$$

and satisfies

$$
\theta_{s}=\kappa,
$$

obtained from the Frenet-Seret relations. We may integrate the above equations, using $\mathrm{d} \mathbf{X} / \mathrm{d} s=\hat{\mathbf{s}}$, to determine the shape of $\Gamma$ from $\theta$. Note that $\theta$ is $2 \pi$-periodic modulo $2 \pi$ with respect to $\alpha$. For $\Gamma$ a closed loop with no self-intersections, we have the condition $\theta(\alpha+2 \pi)=\theta(\alpha)+2 \pi$.

From Eq. (8), we obtain

$$
\theta_{t}=\hat{\mathbf{n}} \cdot \mathbf{V}_{s}
$$

and inserting Eqs. (4) and (16), we obtain

$$
\begin{aligned}
\bar{\mu} \theta_{t}= & -\left(M_{0}(y)+2\right) \theta_{s s s}+2 M_{0}(y)\left(\theta_{s} T_{s}+\theta_{s}^{2} \theta_{s s}\right)+\left(M_{0}(y)+2\right)\left(\theta_{s} T\right)_{s} \\
& +\hat{\mathbf{n}} \cdot \partial_{s} \mathbf{K}\left[\left(T_{s}+\theta_{s} \theta_{s s}\right) \hat{\mathbf{s}}+\left(\theta_{s} T-\theta_{s s s}\right) \hat{\mathbf{n}}\right],
\end{aligned}
$$

which is nonlinear in $\theta$, but linear in $T$. The highest-order term is fourth-order, arising from the filament elasticity. Tension $T$ here, determined from Eq. (14), constrains $\theta$ such that Eq. (5) is satisfied. Finally, integrating Eq. (18) in time determines the time-evolution of the centerline $\Gamma$.

Some additional comments are:

1. No external flow sources are included, although it is possible to incorporate such effects (see [19]).

2. As a simplification, we eliminate free ends by making the filaments closed loops. This need not be considered as an artificial constraint — such a loop is observed experimentally in [22]. Johnson [18] shows that corrections to the force-velocity relation (3) for free ends may be determined through the introduction of the higher-order Stokeslets of Chwang and $\mathrm{Wu}[5]$.

3. Thermodynamical effects are neglected, beyond specifying parameter values $\sigma$ and $\bar{\mu}$ as in the work of $\mathrm{E}$ and Palffy-Muhoray [7].

4. Note that the non-local dynamics of an inextensible filament are gotten by setting $\sigma=0$ in Eq. (5), which in turn sets $s_{\alpha t} / s_{\alpha}=0$ in Eq. (14), the integro-differential equation for $T$. 

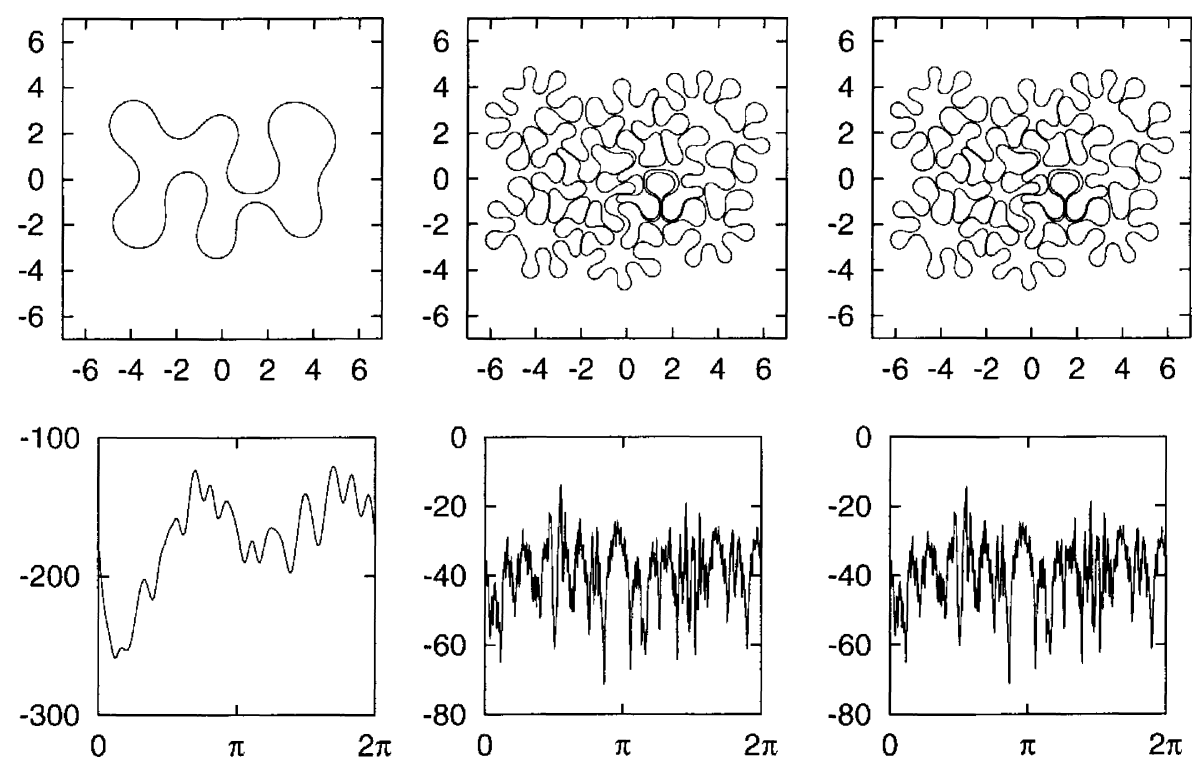

Fig. 3. Numerical simulations with and without interpolation. Top row gives the filament profile, bottom row, the line tension. Left column at $t=0.0$, middle and right column at $t=1.6$. Middle column with $N=2048$ discretization points and interpolation, right column with $N=4096$ and no interpolation. Run parameters $\bar{\mu} / c_{\circ}=65, a=0.02$, and time step size $\Delta t=2.5 \times 10^{-4}$. Not shown are results with $N=2048$ and no interpolation, which self-intersected at $t=1.2$ due to a lack of resolution.

\subsection{Numerical methods}

While details on the numerical methods, we use to simulate the filament equations, are given in Appendix D, we outline some of the main numerical issues here. We use a uniform discretization in $\alpha$ and obtain spectral accuracy by evaluating $\alpha$ derivatives pseudo-spectrally using discrete Fourier transforms (DFTs). To avoid numerical stiffness arising from the fourth-order elasticity term in Eq. (18), we apply the small-scale decomposition approach of Hou et al. [17] and the linear propagator method [24]. These methods allow the small-scale behavior, which is dominated by the elastic regularization, to be handled exactly in DFT space. A two-step second-order Adams-Bashforth method is used for time integration, where the tension $T$ is solved at each time step using GMRES $[3,26]$ using a spectral preconditioning.

When segments of the filament are deemed to have come sufficiently close (as would happen on self-approach), a fixed number of additional points are interpolated between the discretization points for the integral kernel of Eq. (11) near the peaks of $1 /|\mathbf{R}|$. This allows us to extend our simulations to longer times without an undue increase in the total number of discretization points. With the modified quadrature, the inclusion of these interpolated points leads to our code being second-order accurate in space. Fig. 3 compares a simulational run with interpolations to a run without interpolations, but with double the number of discretization points, showing good correspondence.

\section{Buckling instability and pattern formation}

\subsection{Linearized analyses}

Both the local and non-local drag models display buckling instabilities. Since they are similar, we first elucidate the basic mechanisms of the instabilities through the local drag model. It will then be compared to the analogous analyses for the non-local drag model. 
Retaining only the logarithmic leading-order terms in Eqs. (14) and (18), we obtain

$$
\begin{aligned}
& 2 T_{s s}-\left(\theta_{s}\right)^{2} T=\tilde{\mu}-2\left(\theta_{s s}\right)^{2}-3 \theta_{s} \theta_{s s s}, \\
& \tilde{\mu} \theta_{t}=-\theta_{s s s s}+\left(2\left(\theta_{s}\right)^{2}+T\right) \theta_{s s}+3 \theta_{s} T_{s},
\end{aligned}
$$

where $\tilde{\mu}=\bar{\mu} / M_{0}(y)$. Here, we scale viscosity such that $\tilde{\mu}=\mathrm{O}(1)$. Flexing arises from the coupling of the fourth-order elastic regularization and the (anti-)diffusive terms of Eq. (20). In effect, there is an anti-diffusive term if a negative tension $T$ becomes less than $2 \kappa^{2}$.

Numerical simulations of the local drag model with free ends, stipulating boundary conditions $\kappa=\kappa_{s}=0$ and $T=0$ at the ends, suggest a critical length beyond which the filament centerline buckles, inducing non-zero curvatures throughout its length. The growth of the filament causes the radius of curvature of the bends to increase with time. After this initial buckling from the straight configuration, we conjecture a critical radius of curvature, beyond which further buckling occurs.

To obtain such a critical radius, let us study the simple case where $\Gamma$ is initially a circular loop. This admits the solution

$$
T_{\circ}=-\tilde{\mu} \mathrm{e}^{2 t} \quad \text { and } \quad \theta_{\circ}=\alpha,
$$

where we recall $s=\alpha \mathrm{e}^{t}$. The circular shape is preserved and expands outwards exponentially with time. The tension is negative (compressive) and its magnitude increases exponentially with time. We take the above solution to be the basic state, about which we carry out a linearized stability analysis. Of interest here is determining a critical radius for the circular loop beyond which perturbations to the shape become unstable. Note that, with our non-dimensionalization, the radius for the loop is selected by specifying the value for the initial time $t$.

We perturb the circular loop with a $k$ th Fourier mode of magnitude $\delta(t)$,

$$
\begin{aligned}
& \theta=\theta_{\circ}+\delta \frac{k^{2}-1}{k} \sin (k \alpha)+\mathrm{O}\left(\delta^{2}\right), \\
& T=T_{\circ}+\delta \mathrm{e}^{-2 t} T_{k} \cos (k \alpha)+\mathrm{O}\left(\delta^{2}\right),
\end{aligned}
$$

where $T_{k}$ is a constant and $0<|\delta| \ll 1$. We define the instantaneous growth rate of the perturbative mode by $\sigma_{k}=\delta_{t} / \delta$, which we determine through a linearization of Eqs. (19) and (20),

$$
\sigma_{k}=\frac{k^{2}}{2 k^{2}+1}\left(\left(2 k^{2}-5\right)-\frac{2\left(k^{2}-1\right)^{2}}{\tilde{\mu} \mathrm{e}^{4 t}}\right)
$$

At sufficiently small $t$, and thus for a sufficiently short loop radius, all perturbation modes would be stable. As time and radius increases beyond a critical value, there appears a band of unstable modes with wavenumber $k \in\left[2, k_{\max }\right]$, where the $k=2$ mode is the first to destabilize. Any $k$ mode eventually goes unstable, given sufficient time (in fact, Eq. (24) may be integrated to explicitly solve for $\delta$, which shows that if a mode begins to grow, it grows forever). The critical curvature is determined from when the $k=2$ mode becomes unstable,

$$
\kappa_{b}^{L}=\left(\frac{1}{6} \tilde{\mu}\right)^{1 / 4},
$$

as computed from Eq. (24) [29]. This also determines a critical logarithmic leading-order tension,

$$
T_{\circ}^{L}=-\sqrt{6} \tilde{\mu} .
$$

We present an analogous linearized analysis for our non-local drag model of Eqs. (14) and (18) in Appendix C. As with the local model, there is a critical radius beyond which a band of unstable modes appear, where the first buckling 


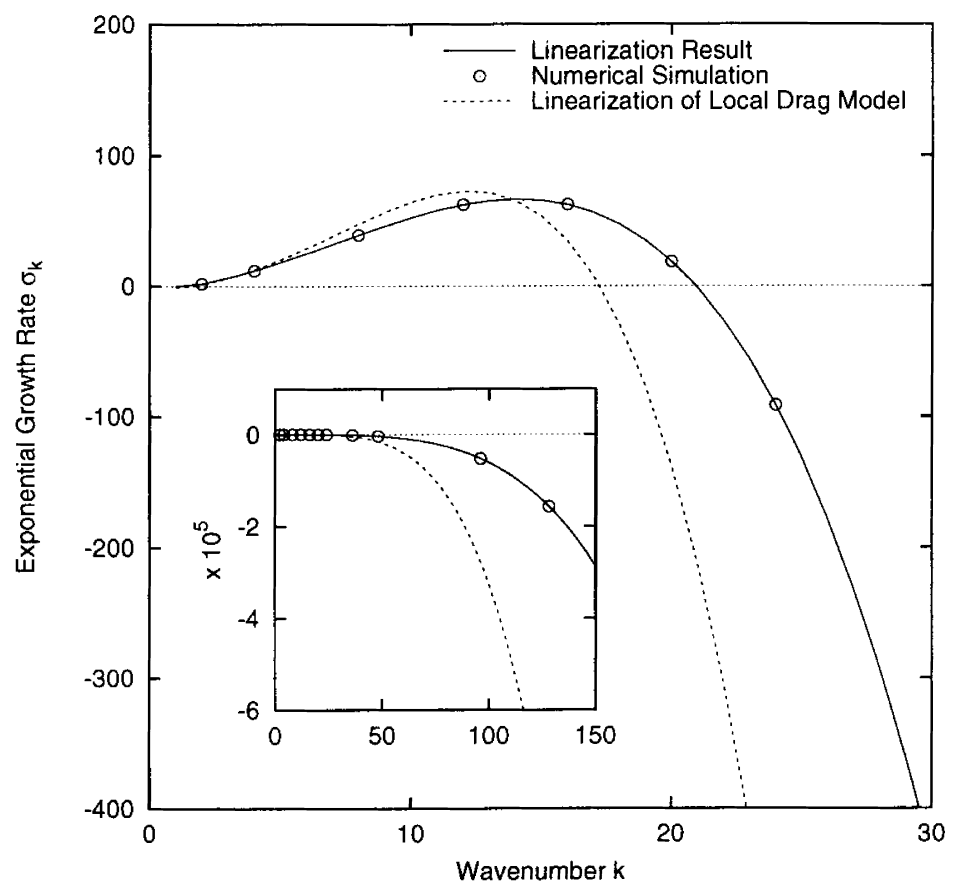

Fig. 4. Exponential growth rate $\sigma_{k}$ of $k$ th wavenumber mode perturbation to a circular loop, for (non-dimensional) filament radius $a=0.01$ and effective viscosity $\bar{\mu} / c_{\circ}=256$. Inset illustrates the stability of the higher-wavenumber modes.

mode is $k=2$. A sample stability diagram is shown in Fig. 4, comparing $\sigma_{k}$ from both the local and non-local models. The differences between the two curves are due to the slow asymptotic convergence of the logarithmic leading-order. It is seen that the unstable bandwidth and the fastest-growing modes are underestimated by the local drag model.

As a consistency check, we plot the growth rates as computed from numerical simulations of our non-local drag model. In these simulations, a perturbed circular loop is allowed to grow for a very short time, and the short-time growth of each Fourier mode is computed through discrete Fourier transforms. We observe a good correspondence between the simulations and the linearization results.

In Fig. 5, we plot the critical curvature $\kappa_{b}$ as determined from the linearized stability analyses versus $\bar{\mu}$ for various values of the filament radius $a$. We observe that, for larger $a$, the relationship between $\kappa_{b}$ and $\bar{\mu}$ strays from the $\frac{1}{4}$-power relationship as given by the local drag model. Indeed, for $a=0.02$, we have $\kappa_{b} \approx 0.754 \bar{\mu}^{0.279}$ from least-squares fitting.

\subsection{Nonlinear simulations}

Fig. 6 gives a sample comparison between the filament evolution over moderate times as simulated from the local and non-local drag models, using identical initial conditions and run parameters. There are observable differences between the patterns for the two models, with the non-local drag model being characterized by a shorter radius of curvature. Because of the slow-convergence, although the aspect ratio is of size $\epsilon \approx 0.0032 \mathrm{e}^{-t}$, the logarithmic order of correction is of size $1 / \log (1 / \epsilon) \approx 1 /(5.7+t)$.

The critical radius and tension as determined from the first buckling instability ( $k=2$ mode) of the linearization analysis characterizes the length-scale of the labyrinthine pattern and the filament tension. In Fig. 7, arclength 


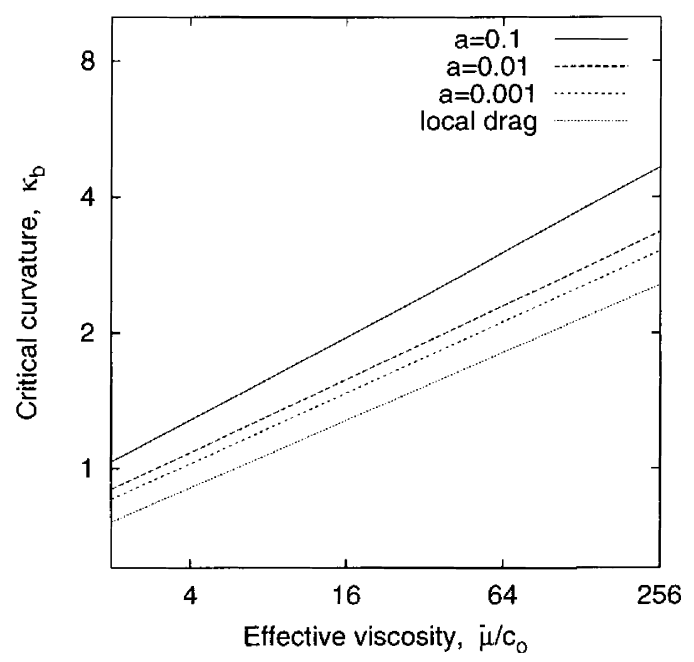

Fig. 5. Critical curvature $\kappa_{b}$ for onset of $k=2$ instability for various values of the filament radius $a$ from the linearized analysis compared with the local drag model result of Eq. (25).

curvatures corresponding to plots in Fig. 2 are compared with $\kappa_{b} \approx \pm 2.4$ as determined from the linearization. We observe that the extrema in the curvature plots extend vertically with time, until reaching a value approximately given by $\kappa_{b}$. At that point, the first buckling instability takes place, and an extremum is seen to "tip split" into three, of which the middle one extends vertically in the opposite direction, until it approximately reaches $\kappa_{b}$ with the opposite sign. Note that, for this run, the local model linearization underestimates the characteristic curvature, $\kappa_{b}^{L} \approx \pm 1.81$.

Fig. 8 displays the line tensions corresponding to the time snapshots given in Fig. 2, plotted with the critical value as computed from the linearization. The filament is continuously being driven to buckle. A bend in the filament expands outwards, increasing the radius of curvature. This in turn increases the magnitude of the negative tension, until the buckling instability both relieves the local compression and decreases the local radius of curvature. This system lives on a marginal stability boundary, where repeated bucklings drives the flexing, leading eventually to a space-filling, labyrinthine pattern.
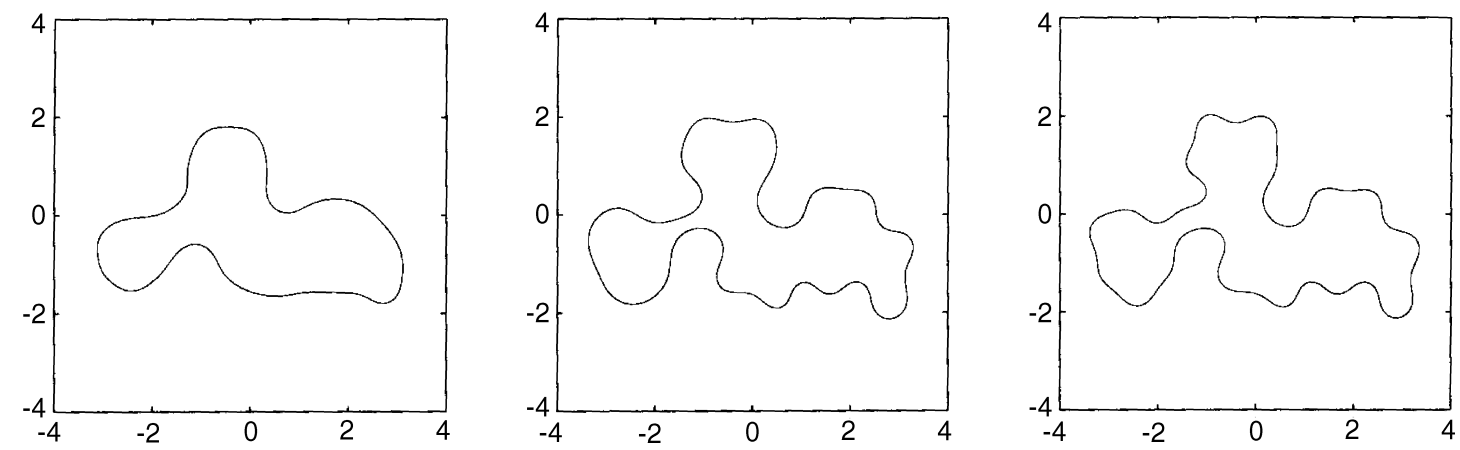

Fig. 6. From left to right, the filament curve at $t=0.0$, the filament evolved by the local model at $t=0.3$ and by our non-local model at $t=0.3$. Filament radius $a=0.02$ and effective viscosity $\bar{\mu} / c_{\circ}=65$. 

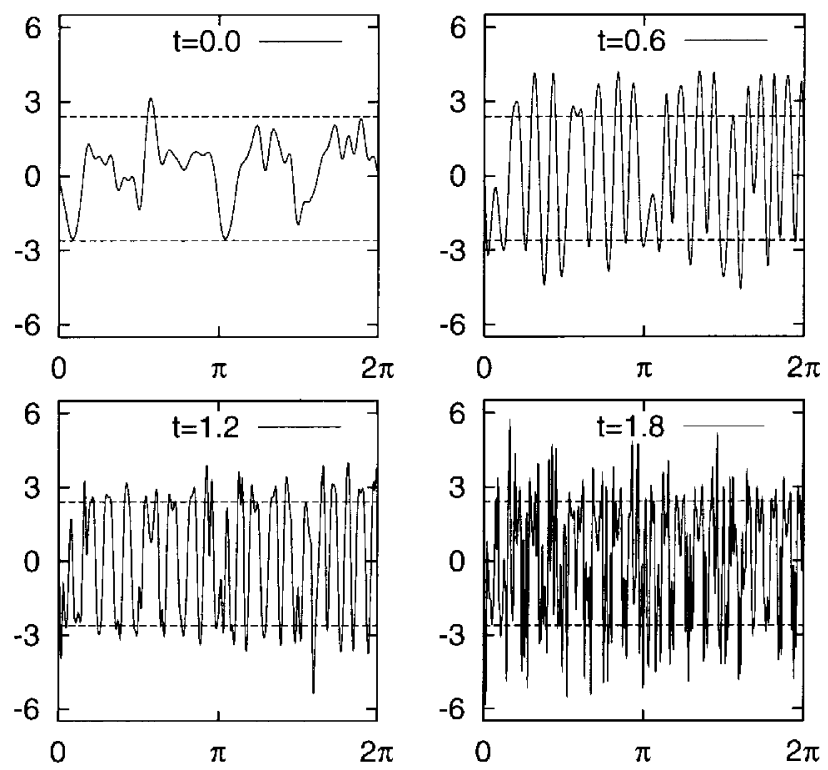

Fig. 7. Time snapshots of arclength curvature $\kappa$ versus Lagrangian $\alpha$ corresponding to the plots in Fig. 2 compared with the characteristic curvature $\left|\kappa_{b}\right| \approx 2.4$ from the linearized stability analysis. Filament radius $a=0.02$ and effective viscosity $\bar{\mu} / c_{\circ}=65$.
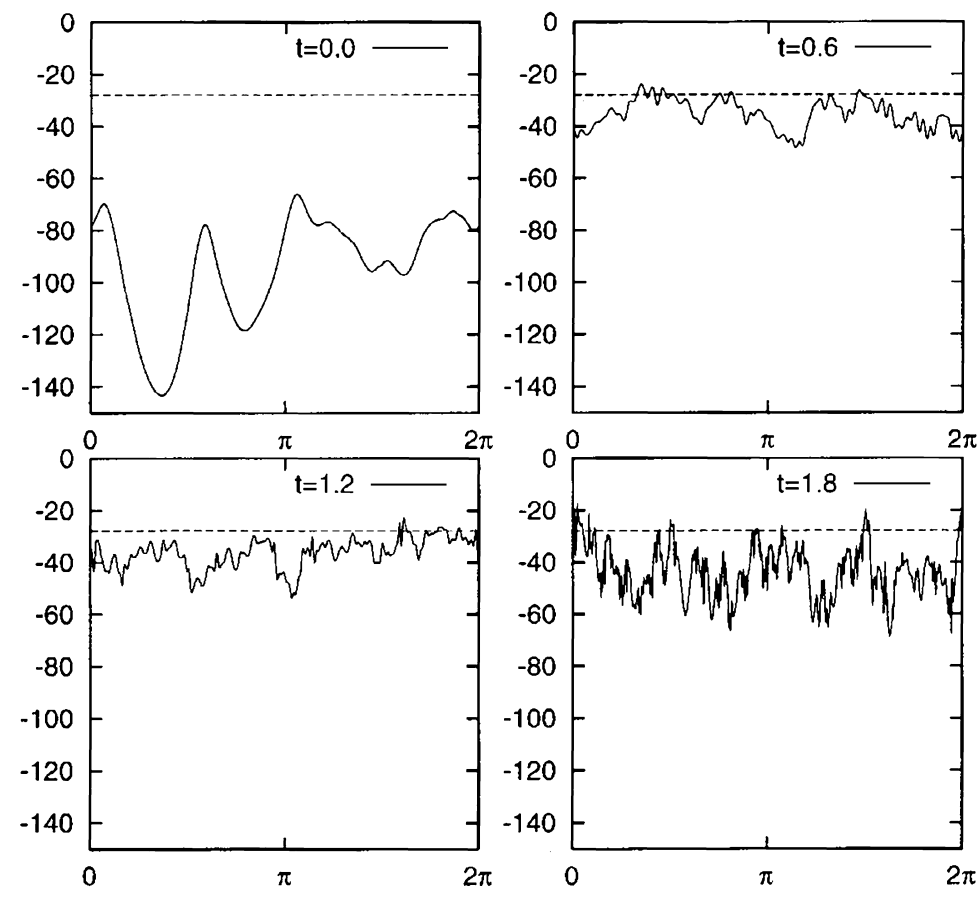

Fig. 8. Time snapshots of tension $T$ versus Lagrangian $\alpha$ corresponding to the plots in Fig. 2 compared with critical tension $T_{\circ} \approx-27.9$ from the linearization. Filament radius $a=0.02$ and effective viscosity $\bar{\mu} / c_{\circ}=65$. 


\section{Filament packing and the effects of viscosity}

Growth and flex leads to self-approach, as separated segments of the filament are driven towards one another. We have shown previously [29] that unphysical self-intersections of the filament centerline are observed for the local model (there are, in fact, purely local models, such as the Gage-Hamilton model [9,15] for flow by curvature, which disallow self-intersections). With our non-local model, however, the kernel of Eq. (11) becomes near-singular as $|\mathbf{R}| \ll 1$ along points of self-approach, scaling logarithmically with the separation distance. Self-intersections would lead to singularities in the integral kernel.

Numerical simulations suggest that this is sufficient to prevent self-intersections. Fig. 9 shows snapshots from two such simulations, with different values for the effective viscosity. No self-crossings are observed, for these or for other simulations which we have carried out. In Fig. 10, we magnify the central regions of the top row of Fig. 9. On approach, the segments flatten and align to become near-parallel. By marking locations along the centerline with fixed arclength coordinates, we observe that, although the relative transverse motions appear halted, the segments may slide past one another.

Fig. 11 shows the minimum distance between the two approaching filament segments centered in Fig. 10. This distance is computed from the discretized $\mathbf{X}$ data using DFT interpolations. Results with increasing numbers of discretization points (as decreasing arclength distance between the points) are compared to safeguard against any numerical discretization errors. The segments approach with near-constant speed (note that Fig. 11 has a logarithmic vertical scale), then rapidly decelerate, rebound, and then settle down to what appears to be a constant minimum separation distance. This rapid deceleration is consistent with the scaling of the logarithmic near-singularity of the integral kernel.

The computed value for the minimum approach distance is unphysical, however, since at times it falls below twice the filament radius $a$. Note that, in our inner-outer matched asymptotic formulation, filament segments were assumed to be separated on the outer $L_{\circ}$ length-scale. Any approach closer than that would be beyond the resolution
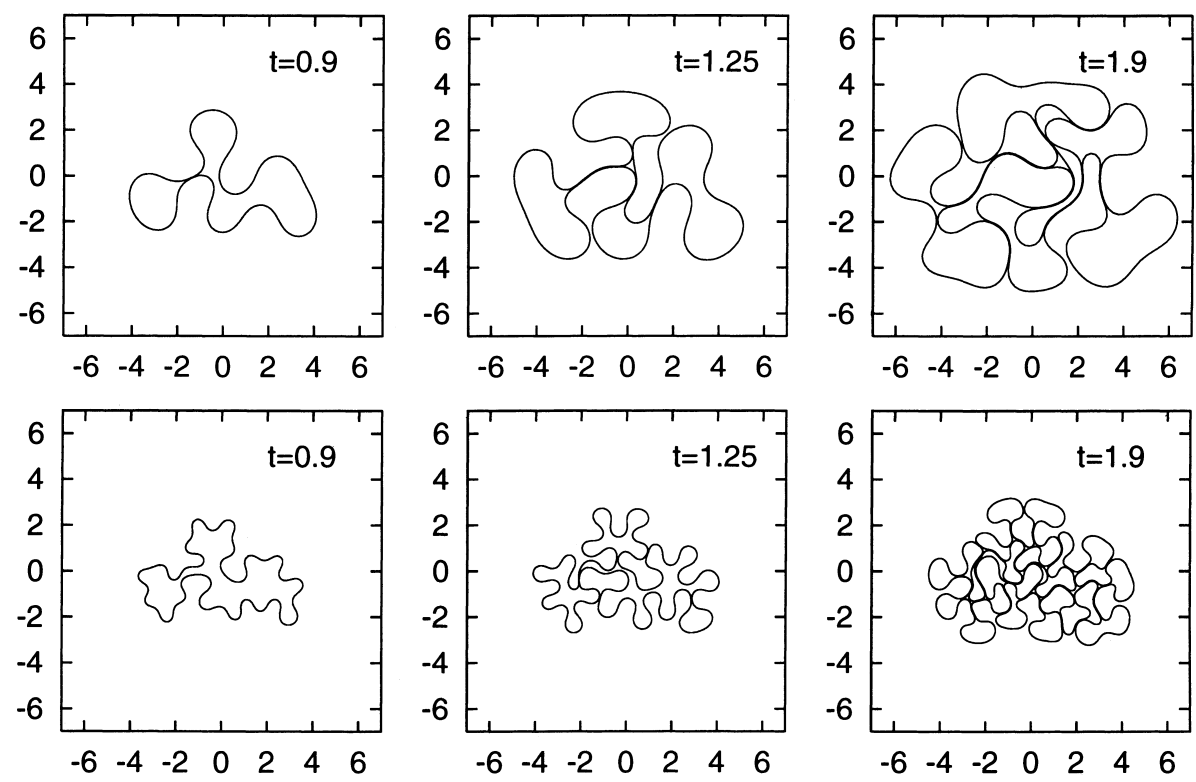

Fig. 9. Filament curves with different effective viscosities. Snapshots at time $t=0.6,1.25$, and 1.9. The top row with $\bar{\mu} / c_{\circ}=2$ and the bottom row with $\bar{\mu} / c_{\circ}=65$. Other physical parameters and the initial profile as in Fig. 6. 

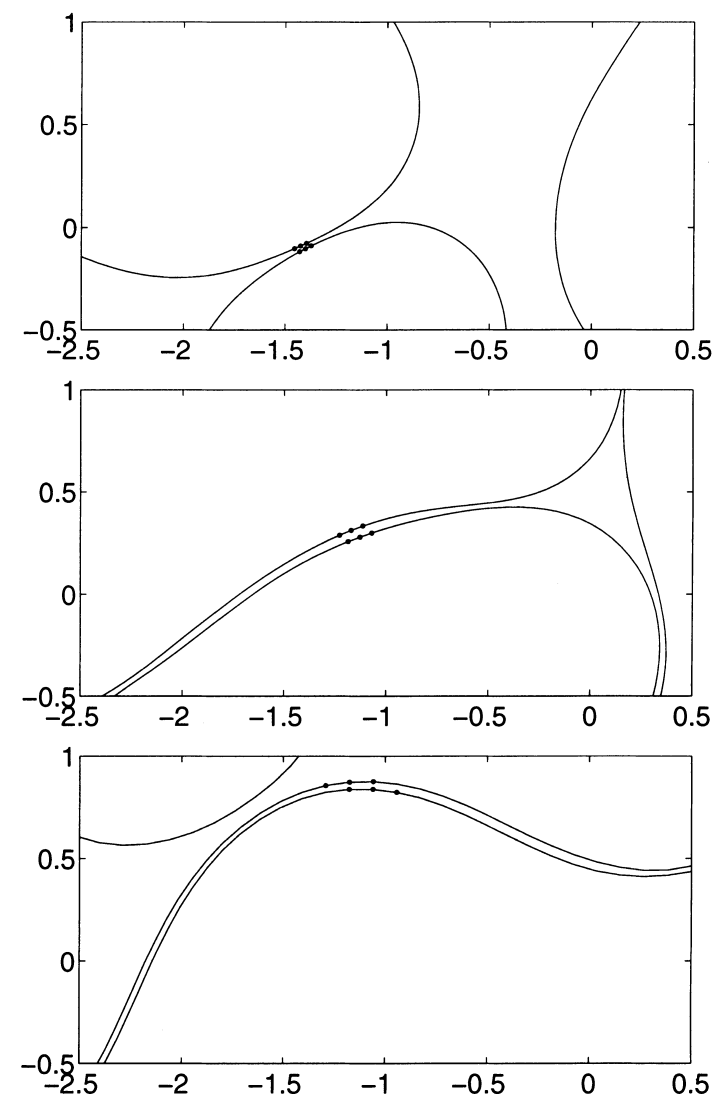

Fig. 10. Close-ups of the plots given in the top row of Fig. 9, showing the approach and separation of filament segments. Six points along the centerline are tracked to illustrate the filament growth and the segments sliding relative to one another.

of our model. It may be possible to add higher-order Stokeslets to the outer solution and refine our inner solution to accurately compute the approach distance, but we have not yet done so. Nevertheless, as a leading-order result, our model remains consistent with the experimental observations in exhibiting an avoidance of self-intersections.

As can be observed from Fig. 9, the centerline curve displays different behaviors in the inner and peripheral regions. Self-approach in the inner region severely restricts any transverse motion, with flexing segments becoming "packed in". The curve shape appears frozen in that region, with sizes of the loops scaling with the characteristic radius $r_{b}=1 / \kappa_{b}$. There is, however, still axial growth and motion, with segments of the filament sliding past one another. On the periphery, segments are free to expand outwards, driven by the growth and the flex, leading to a space-filling curve. This is consistent with the experimental observations of Palffy-Muhoray et al. [23] that "As the filament grows and buckles, it eventually occupies an approximately disk-shaped region in the cell. The filaments grow more rapidly near the edge of this region than close to the center" (they could not directly observe the axial growth, and inferred the filament growth from the transverse motion).

In the interior region, the congestion leads to a squashing of the filament, with segments being alternatively flattened and more sharply bent. With increased packing, this causes larger variations in the curvature from $\kappa_{b}$ as predicted by the linearization. This is illustrated in Fig. 12, which plots the arclength curvatures corresponding to Fig. 9. 


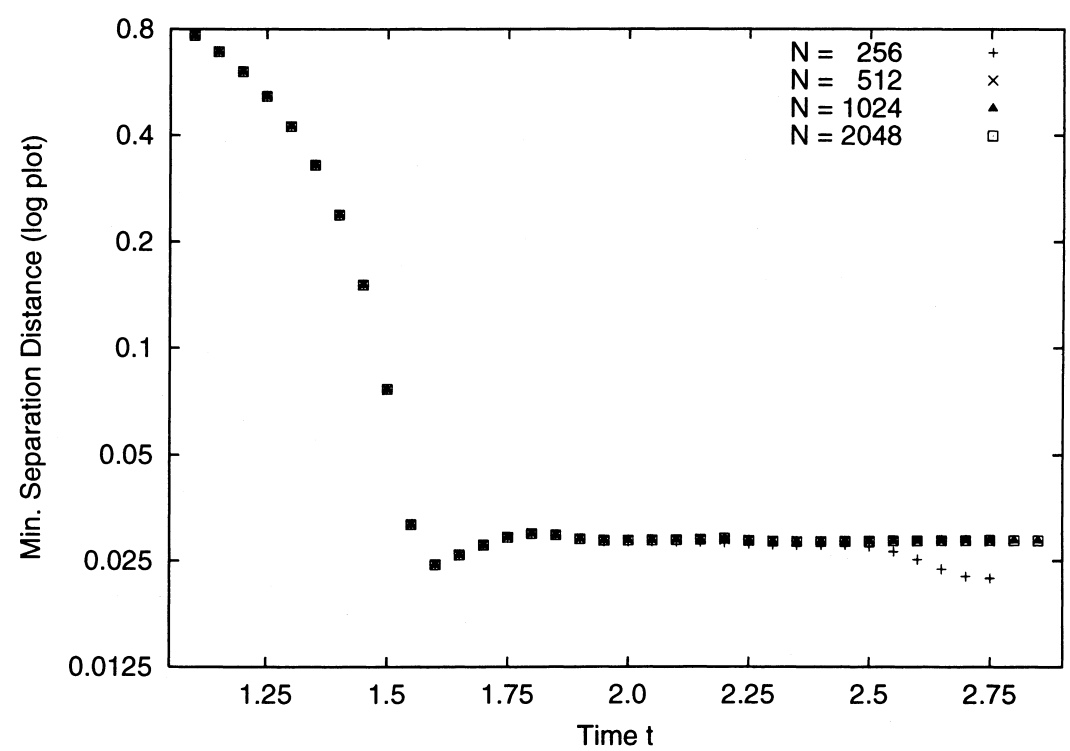

Fig. 11. Log plot of the minimal separation distance along the filament segments of first approach versus time. Numerical simulations with $N$ discretization points and cubic spline interpolation. Run parameters are $\bar{\mu} / c_{\circ}=2, a=0.02, \Delta t=6.25 \times 10^{-5}, N_{I}=12$ and $n_{I}=6$.
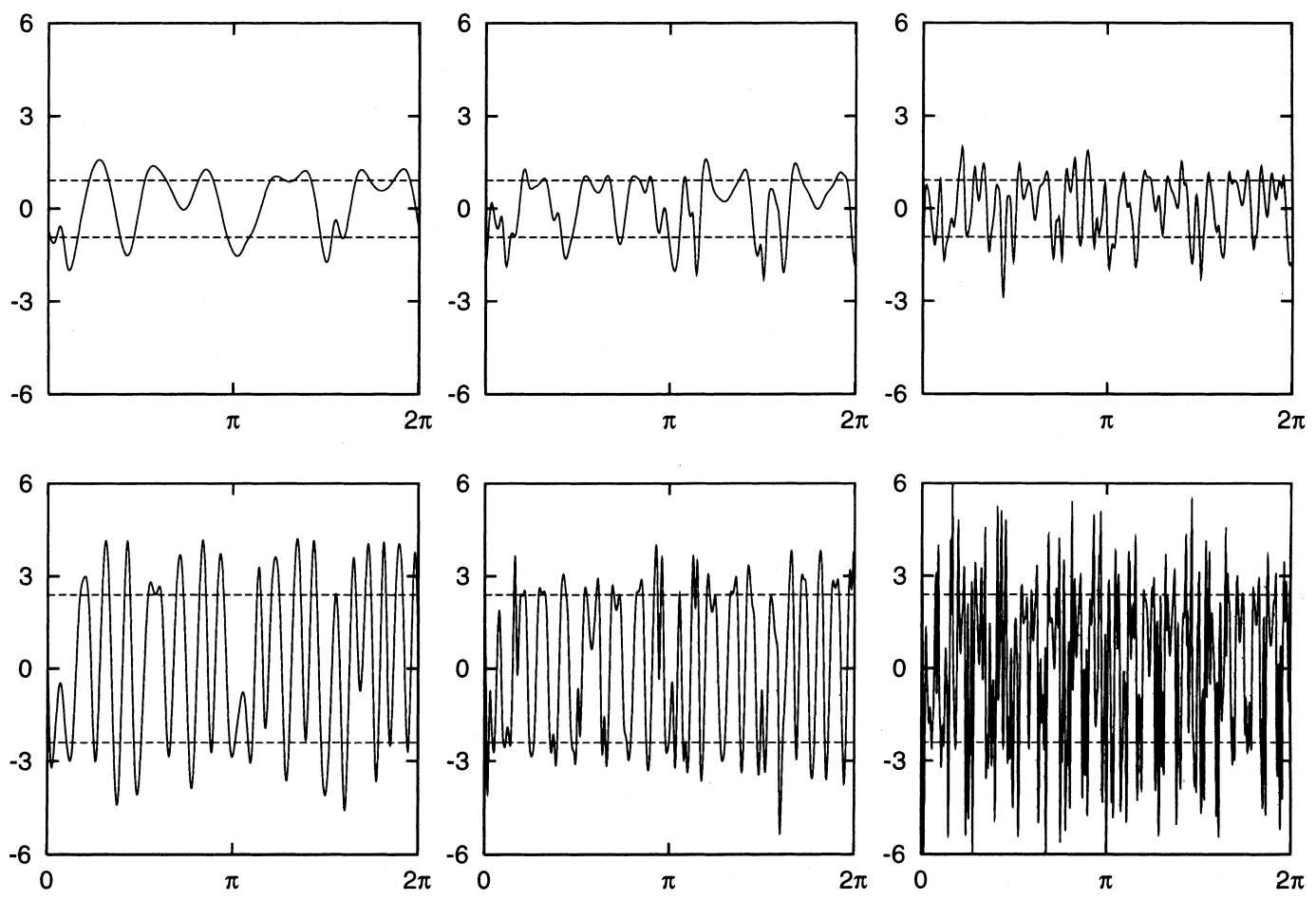

Fig. 12. Filament curvature versus Lagrangian $\alpha$ with different effective viscosities corresponding to snapshots in Fig. 9. Also plotted are the characteristic curvatures as suggested by the linearization, $\kappa_{b} \approx 0.92$ for $\bar{\mu} / c=2$ and $\kappa_{b} \approx 2.4$ for $\bar{\mu} / c=65$. 
The region occupied by the filament expands outwards, driven by the flexing of the filament segments on the outer periphery. In this, it is reminiscent of a fingering instability as may be seen, e.g., in Hele-Shaw cells. The rate of expansion into the outer region scales with the characteristic radius $r_{b}$. Thus, we see in Fig. 9 that the larger effective viscosity leads to a slower expansion.

\section{Concluding remarks}

In this paper, we study the motion of a filament immersed in Stokes hydrodynamics. Dynamics of the filament and fluid flow is intimately coupled, with the filament growth driving the flow and the flow deforming the filament shape. Our integro-differential model does not suffer from asymptotic slow convergence, and does not exhibit unphysical higher-wavenumber instabilities. Our numerical scheme has high spatial accuracy and is not constrained by stiffness. Numerical simulations show that our model captures the global dynamics of the filament motion, and results in flexing patterns very similar to those observed in experiments of the $I-S_{A}$ phase transition.

There are several natural directions in which this work could be extended. First, although the fluid flow is fully three-dimensional, our current filament simulations are constrained to two dimensions. With fully three-dimensional motion, the effect of filament torsion and twist could be included in the non-local hydrodynamics (though this may not be an important effect for the smectic-A filament problem). There has been work in this direction. For example, in a study motivated by observations of shape instabilities of mutant strains of bacteria [21], Goldstein et al. [11] have considered the overdamped dynamics of a three-dimensional, inextensible filament with bend and twist elasticity, using an isotropic local damping model. Goriely and Tabor [12,13] have studied three-dimensional dynamics and stability of elastica moving solely under their own inertia. In both of these studies, a central question is the choice of a natural three-dimensional frame in which to study the dynamics.

Further, the hydrodynamics here are driven purely through the filament motion, and there are no imposed external flows (such as shear), external walls, or other filaments. By including such additional effects, it may be possible to model more complex fluids, such as the flow of dilute polymeric fluids. Third, the separation distance on filament self-approach cannot be resolved to the short inner length-scale (our current model, however, does prevent self-crossings of the filament and thus captures that aspect of the global dynamics). For resolution to such short scales near where the filament is self-approaching, a more faithful fluid dynamic description will be required. One approach might be to require higher-order Stokeslets in the outer expansion, and for balance, corrections for the inner expansion, where the filament is considered not as an isolated straight cylinder but as a pair of cylinders, perhaps in near-parallel alignment. Conversely, perhaps the prospect should be explored of simulating (without the advantages and short-comings of a slender-body approximation) the dynamics of fully three-dimensional elastic filaments interacting within a Stokesian fluid.

\section{Acknowledgements}

We extend our thanks to Raymond Goldstein and David Muraki for useful and stimulating conversations about various aspects of this work. We thank especially Peter Palffy-Muhoray for sharing his experimental results and video with us, and Leif Becker for a critical reading and discussions of the paper. MJS acknowledges partial support from DOE grant DE-FG02-88ER25053MJS, NSF grant DMS-9404554 and Presidential Young Investigator grant DMS-9396403. TU acknowledges the support of AFOSR grant AFOSR-900161. 


\section{Appendix A. Asymptotic consistency with Keller and Rubinow}

The Keller and Rubinow force-velocity relation [19] in non-dimensionalized variables is

$$
\begin{aligned}
\bar{\mu} \mathbf{V}(\alpha, t)= & -c \mathbf{A}(\alpha, t) \cdot \mathbf{f}(\alpha, t)-\int_{-\pi}^{\pi}\left[\mathbf{S}\left(\mathbf{R}\left(\alpha, \alpha^{\prime}, t\right)\right) \mathbf{f}\left(\alpha+\alpha^{\prime}, t\right)-\frac{\mathbf{A}(\alpha, t) \cdot \mathbf{f}(\alpha, t)}{s_{\alpha}\left|\alpha^{\prime}\right|}\right] s_{\alpha} \mathrm{d} \alpha \\
& -2 \hat{\mathbf{n}}(\alpha, t)(\hat{\mathbf{n}}(\alpha, t) \cdot \mathbf{f}(\alpha, t))+\mathrm{O}\left(\epsilon^{2} \log \epsilon\right),
\end{aligned}
$$

where $\mathbf{R}\left(\alpha, \alpha^{\prime}, t\right)=\mathbf{X}(\alpha, t)-\mathbf{X}\left(\alpha+\alpha^{\prime}, t\right)$, the coefficient $c=-\log \left(\frac{1}{16} \epsilon^{2}\right.$ e $\left.\pi^{2}\right)$, the tensor $\mathbf{A}=\mathbf{I}+\hat{\mathbf{s}} \hat{\mathbf{s}}$, and the Stokeslet kernel $\mathbf{S}(\mathbf{R})=(\mathbf{I}+\hat{\mathbf{R}} \hat{\mathbf{R}}) /|\mathbf{R}|$ (see [5]). We can use the integral identity

$$
\int_{-\pi}^{\pi}\left(\frac{1}{\left|2 \sin \left(\alpha^{\prime} / 2\right)\right|}-\frac{1}{\left|\alpha^{\prime}\right|}\right) \mathrm{d} \alpha^{\prime}=2 \log \left(\frac{4}{\pi}\right)
$$

to transform the second term of the integrand to a form more amenable to closed loops.

In the limit $0<y \ll 1$, we may re-express our force-velocity relation, Eq. (9), as

$$
\bar{\mu} \mathbf{V}(\alpha, t)=-\int_{-\pi}^{\pi} \frac{\mathbf{I}+\hat{\mathbf{R}}\left(\alpha, \alpha^{\prime}, t\right) \hat{\mathbf{R}}\left(\alpha, \alpha^{\prime}, t\right)}{\left(\left(\left|\mathbf{R}\left(\alpha, \alpha^{\prime}, t\right)\right| / s_{\alpha}\right)^{2}+y^{2}\right)^{1 / 2}} \cdot \mathbf{f}\left(\alpha+\alpha^{\prime}, t\right) \mathrm{d} \alpha^{\prime}-2 \hat{\mathbf{n}}(\alpha, t)(\hat{\mathbf{n}}(\alpha, t) \cdot \mathbf{f}(\alpha, t))+\mathrm{O}\left(y^{2} \log y\right) .
$$

We can explicitly draw out the logarithmic leading-order term by

$$
\begin{aligned}
\bar{\mu} \mathbf{V}(\alpha, t)= & -\mathbf{A}(\alpha, t) \cdot \mathbf{f}(\alpha, t) M_{0}(y) \\
& -\int_{-\pi}^{\pi}\left[\frac{\left(\mathbf{I}+\hat{\mathbf{R}}\left(\alpha, \alpha^{\prime}, t\right) \hat{\mathbf{R}}\left(\alpha, \alpha^{\prime}, t\right)\right) \cdot \mathbf{f}\left(\alpha+\alpha^{\prime}, t\right)}{\left(\left(\left|\mathbf{R}\left(\alpha, \alpha^{\prime}, t\right)\right| / s_{\alpha}\right)^{2}+y^{2}\right)^{1 / 2}}-\frac{\mathbf{A}(\alpha, t) \cdot \mathbf{f}(\alpha, t)}{\left(4 \sin ^{2}\left(\alpha^{\prime} / 2\right)+y^{2}\right)^{1 / 2}}\right] \mathrm{d} \alpha^{\prime} \\
& -2 \hat{\mathbf{n}}(\alpha, t)(\hat{\mathbf{n}}(\alpha, t) \cdot \mathbf{f}(\alpha, t)),
\end{aligned}
$$

which is Eq. (A.2) rewritten exactly. Since

$$
M_{0}(y)=2 \log \left(\frac{8}{y}\right)+\mathrm{O}\left(y^{2} \log y\right) \text { for } 0<y \ll 1
$$

(see [14, Eqs. (8.11) and (8.128)]), the first terms of Eqs. (A.1) and (A.3) are asymptotically equivalent once we specify $y=2 \pi \sqrt{\mathrm{e}} \epsilon$.

It is now left to show the asymptotic equivalence of the integral terms. Fixing time $t$, the integral takes the form

$$
I(\epsilon)=\int_{-1}^{1} \mathrm{~d} s\left[\frac{g(s)}{\left(|\mathbf{R}(s)|^{2}+\epsilon^{2}\right)^{1 / 2}}-\frac{g(0)}{\left(s^{2}+\epsilon^{2}\right)^{1 / 2}}\right]
$$

for $0<\epsilon \ll 1$, and where $\mathbf{R}(0)=\mathbf{0}$, and $\mathbf{R}(s)$ gives the position of a planar curve $\Gamma$ relative to the origin. We assume that $\mathbf{R}(s)$ and $g(s)$ are smooth in $s$, and also that $\Gamma$ is strictly bounded away from any self-intersections. We then have the following required expansion:

$$
I(\epsilon)=\int_{-1}^{1} \mathrm{~d} s\left[\frac{g(s)}{|\mathbf{R}(s)|}-\frac{g(0)}{|s|}\right]+2 h_{2} \epsilon^{2} \ln \epsilon^{2}+\mathrm{O}\left(\epsilon^{2}\right)
$$

for $\epsilon$ sufficiently small, and where $h_{2}=\frac{1}{2} g_{0}^{\prime \prime}-\frac{1}{8} \kappa_{0}^{2} g_{0}$. The subscript 0 refers to evaluation at the origin, $\kappa$ is the curvature of $\Gamma$, and prime denotes a derivative with respect to $s$. 
Proof. We set $r(s)=\operatorname{sgn}(s)|\mathbf{R}(s)|$, i.e., the signed distance from the origin. Expanding near $s=0$, and using the Frenet-Seret formulae, we have

$$
\begin{aligned}
\mathbf{R}(s) & =\hat{\mathbf{s}}_{0} s+\frac{1}{2} \kappa_{0} \hat{\mathbf{n}}_{0} s^{2}+\frac{1}{6}\left(\kappa_{0}^{\prime} \hat{\mathbf{n}}_{0}-\kappa_{0}^{2} \hat{\mathbf{s}}_{0}\right) s^{3}+\mathrm{O}\left(s^{4}\right), \\
r(s) & =s-\frac{1}{24} \kappa_{0}^{2} s^{3}+\mathrm{O}\left(s^{4}\right), \quad r^{\prime}(s)=1-\frac{1}{8} \kappa_{0}^{2} s^{2}+\mathrm{O}\left(s^{3}\right) .
\end{aligned}
$$

These expansions will be used to exchange a dependence on $s$ with a dependence on $r$ in the first term of Eq. (A.5). Expanding $g(s)$ around the origin, and using the above expansions yields

$$
\begin{aligned}
g(s)=g_{0}+g_{0}^{\prime} s+\frac{1}{2} g_{0}^{\prime \prime} s^{2}+\mathrm{O}\left(s^{3}\right) & =\left(g_{0}+g_{0}^{\prime} r(s)+\left(\frac{1}{2} g_{0}^{\prime \prime}+\frac{1}{8} \kappa_{0}^{2} g_{0}\right) r^{2}(s)\right) r^{\prime}(s)+\mathrm{O}\left(s^{3}\right) \\
& =\left(g_{0}+h_{1} r+h_{2} r^{2}\right) r^{\prime}(s)+\mathrm{O}\left(s^{3}\right)=H(r) r^{\prime}(s)+\mathrm{O}\left(s^{3}\right) .
\end{aligned}
$$

For $s \geq 0$, the change of variables from $s$ to $r$ can be accomplished if $r^{\prime}(s)=(\mathbf{R} /|\mathbf{R}|) \cdot \mathbf{R}^{\prime} \geq C>0$ for some constant $C$. This will be the case in some non-empty interval $[0, \bar{s}]$, where $\bar{s}$ is independent of $\epsilon$. The integral $I$ is now written as $I^{+}+I^{-}$, where $I^{+}$is the integrand integrated on $[0,1]$, and $I^{-}$is likewise the integrand integrated on $[-1,0]$. We can write $I^{+}$as

$$
\begin{aligned}
I^{+}(\epsilon)= & \int_{0}^{1} \mathrm{~d} s \frac{g(s)-H(r(s)) r^{\prime}(s)}{\left(r^{2}(s)+\epsilon^{2}\right)^{1 / 2}}+\int_{0}^{\bar{s}} \mathrm{~d} s\left(\frac{H(r(s)) r^{\prime}(s)}{\left(r^{2}(s)+\epsilon^{2}\right)^{1 / 2}}-\frac{g_{0}}{\left(s^{2}+\epsilon^{2}\right)^{1 / 2}}\right) \\
& +\int_{\bar{s}}^{1} \mathrm{~d} s\left(\frac{H(r(s)) r^{\prime}(s)}{\left(r^{2}(s)+\epsilon^{2}\right)^{1 / 2}}-\frac{g_{0}}{\left(s^{2}+\epsilon^{2}\right)^{1 / 2}}\right)=I_{1}^{+}+I_{2}^{+}+I_{3}^{+} .
\end{aligned}
$$

We now provide estimates for each integral separately, leaving $I_{2}^{+}$for last.

1. Note that by construction the numerator of $I_{1}^{+}$has a third-order zero at $s=0$. One can show that this makes the integrand of sufficient smoothness that one can find the bound

$$
\left|\int_{0}^{1} \mathrm{~d} s \frac{g(s)-H(r(s)) r^{\prime}(s)}{\left(r^{2}(s)+\epsilon^{2}\right)^{1 / 2}}-\int_{0}^{1} \mathrm{~d} s \frac{g(s)-H(r(s)) r^{\prime}(s)}{r(s)}\right|<C_{1} \epsilon^{2}
$$

for $\epsilon$ sufficiently small, and $C_{1}$ a constant.

2. Since $\bar{s}$ is independent of $\epsilon$, and $\Gamma$ is strictly bounded away from self-intersection, $I_{3}^{+}$is regular in $\epsilon$, and can be expanded to yield

$$
\left|\int_{\bar{s}}^{1} \mathrm{~d} s\left(\frac{H(r(s)) r^{\prime}(s)}{\left(r^{2}(s)+\epsilon^{2}\right)^{1 / 2}}-\frac{g_{0}}{\left(s^{2}+\epsilon^{2}\right)^{1 / 2}}\right)-\int_{\bar{s}}^{1} \mathrm{~d} s\left(\frac{H(r(s)) r^{\prime}(s)}{r(s)}-\frac{g_{0}}{s}\right)\right|<C_{2} \epsilon^{2}
$$

for $\epsilon$ sufficiently small, and $C_{2}$ a constant.

3. We are left with $I_{2}^{+}$, which can be explicitly evaluated using the three integral identities:

$$
\begin{aligned}
& \int^{y} \mathrm{~d} y \frac{1}{\sqrt{y^{2}+\epsilon^{2}}}=\ln \left(y+\left(y^{2}+\epsilon^{2}\right)^{1 / 2}\right), \quad \int^{y} \mathrm{~d} y \frac{y}{\sqrt{y^{2}+\epsilon^{2}}}=\sqrt{y^{2}+\epsilon^{2}}, \\
& \int^{y} \mathrm{~d} y \frac{y^{2}}{\sqrt{y^{2}+\epsilon^{2}}}=\frac{1}{2}\left(y\left(y^{2}+\epsilon^{2}\right)^{1 / 2}-\epsilon^{2} \ln \left(y+\left(y^{2}+\epsilon^{2}\right)^{1 / 2}\right)\right) .
\end{aligned}
$$


We set $\bar{r}=r(\bar{s})$, and write

$$
\begin{aligned}
I_{2}^{+}= & \int_{0}^{\bar{r}} \mathrm{~d} r \frac{H(r)}{\left(r^{2}+\epsilon^{2}\right)^{1 / 2}}-\int_{0}^{\bar{s}} \mathrm{~d} s \frac{g_{0}}{\left(s^{2}+\epsilon^{2}\right)^{1 / 2}} \\
= & g_{0} \ln \frac{\bar{r}+\left(\bar{r}^{2}+\epsilon^{2}\right)^{1 / 2}}{\bar{s}+\left(\bar{s}^{2}+\epsilon^{2}\right)^{1 / 2}}+h_{1}\left(\left(\bar{r}^{2}+\epsilon^{2}\right)^{1 / 2}-\epsilon\right) \\
& +\frac{h_{2}}{2}\left(\bar{r}\left(\bar{r}^{2}+\epsilon^{2}\right)^{1 / 2}-\epsilon^{2} \ln \left(\bar{r}+\left(\bar{r}^{2}+\epsilon^{2}\right)^{1 / 2}\right)+\epsilon^{2} \ln \epsilon\right) .
\end{aligned}
$$

Expanding in small $\epsilon$ gives

$$
\begin{aligned}
I_{2}^{+} & =\left(g_{0} \ln \frac{\bar{r}}{\bar{s}}+h_{1} \bar{r}+\frac{1}{2} h_{2} \bar{r}^{2}\right)-h_{1} \epsilon+\frac{h_{2}}{2} \epsilon^{2} \ln \epsilon+\mathrm{O}\left(\epsilon^{2}\right) \\
& =\int_{0}^{\bar{s}} \mathrm{~d} s\left[\frac{H(r(s)) r^{\prime}(s)}{r(s)}-\frac{g_{0}}{s}\right]-h_{1} \epsilon+\frac{h_{2}}{2} \epsilon^{2} \ln \epsilon+\mathrm{O}\left(\epsilon^{2}\right) .
\end{aligned}
$$

Gathering together these three results gives

$$
I^{+}(\epsilon)=\int_{0}^{1} \mathrm{~d} s\left[\frac{g(s)}{r(s)}-\frac{g_{0}}{s}\right]-h_{1} \epsilon+\frac{h_{2}}{2} \epsilon^{2} \ln \epsilon+\mathrm{O}\left(\epsilon^{2}\right),
$$

while a similar calculation for $I^{-}$yields

$$
I^{-}(\epsilon)=\int_{-1}^{0} \mathrm{~d} s\left[\frac{g(s)}{|r(s)|}-\frac{g_{0}}{|s|}\right]+h_{1} \epsilon+\frac{h_{2}}{2} \epsilon^{2} \ln \epsilon+\mathrm{O}\left(\epsilon^{2}\right) .
$$

Upon addition, the $\mathrm{O}(\epsilon)$ terms cancel, yielding the desired result.

\section{Appendix B. High-wavenumber instability of Keller and Rubinow}

Let us conduct a linearized stability analysis of Eq. (A.1) (henceforth referred to as KR), where we perturb about a circular loop. Then $R_{\circ}=2 \exp (t)\left|\sin \left(\alpha^{\prime} / 2\right)\right|$ to leading order and the integral term of KR takes the form

$$
\Psi(\mathbf{g})=\int_{-\pi}^{\pi} \frac{\mathbf{g}\left(\alpha^{\prime}\right)-\mathbf{g}(0)}{2\left|\sin \left(\alpha^{\prime} / 2\right)\right|} \mathrm{d} \alpha^{\prime},
$$

where $\mathbf{g}\left(\alpha^{\prime}\right)=\left(\mathbf{I}+\hat{R}\left(\alpha, \alpha^{\prime}\right) \hat{R}\left(\alpha, \alpha^{\prime}\right)\right) \cdot \mathbf{f}\left(\alpha+\alpha^{\prime}\right)$ is a $2 \pi$-periodic function in $\alpha^{\prime}$. We may write $\mathbf{g}$ in terms of its Fourier series,

$$
\mathbf{g}\left(\alpha^{\prime}\right)=\sum_{k} \mathbf{g}_{k} \mathrm{e}^{\mathrm{i} k \alpha^{\prime}}
$$

and represent the integral as a convolution to obtain

$$
\Psi(\mathbf{g})=-\sum_{k} \mathbf{g}_{k} \Psi_{k}, \quad \text { where } \Psi_{k}=\int_{-\pi}^{\pi} \frac{1-\mathrm{e}^{\mathrm{i} k \alpha^{\prime}}}{2\left|\sin \left(\alpha^{\prime} / 2\right)\right|} \mathrm{d} \alpha^{\prime} .
$$

The weighting factors may be explicitly evaluated to obtain

$$
\Psi_{k}=4 \sum_{j=1}^{k} \frac{1}{2 j-1} \sim 2 \log k \text { for } k \gg 1,
$$

which monotonically increases and diverges logarithmically for large $k$. The logarithmic dependence arises from the jump discontinuity in the integrand of KR. 
The linearized growth rate in the high-wavenumber limit is given by

$$
\sigma_{k}^{\mathrm{KR}} \sim-\frac{k^{4} \mathrm{e}^{-4 t}\left(c-\Psi_{k}\right)}{\bar{\mu}} \text { for } k \gg 1
$$

Since $c \sim-2 \log \epsilon$, at sufficiently high wavenumbers beyond $k \sim 1 / \epsilon$, the modes are unstable. Note that the unstable modes have wavelengths shorter than the filament radius, and so are not resolved in the asymptotically derived KR formula.

Although the KR is asymptotically valid, it is impractical for our purposes here where higher-wavenumber modes grow through the filament flexing. The instability becomes deadly in numerical simulations: finer discretizations would excite the unphysical higher-wavenumber modes through round-off and discretization errors.

\section{Appendix C. Linearized stability analysis}

We present the linearized stability analysis referred to in Section 3. Inserting Eq. (22) into Eq. (9), we obtain the normal and tangential components,

$$
\begin{aligned}
\bar{\mu} s_{\alpha}^{3}(\hat{\mathbf{s}} \cdot \mathbf{V})(\alpha)= & -\delta \sin (k \alpha)\left\{T _ { \circ } \left[\frac{3}{8 k}\left[(k-1)\left(2 k^{2}+4 k+1\right) M_{k+1}-(k+1)\left(2 k^{2}-4 k+1\right) M_{k-1}\right]\right.\right. \\
& \left.+\frac{1}{4 m}\left[\left(3+2 k^{2}\right) M_{k}+3\left(1-2 k^{2}\right) M_{1}+\left(2 k^{2}-3\right) M_{0}\right]-\frac{1}{2} N_{k}\right] \\
& +T_{m}\left[\frac{3}{4}(k+1) M_{k+1}+\frac{3}{4}(k-1) M_{k-1}+\frac{1}{2} k M_{k}\right] \\
& \left.+\frac{1}{4} k\left(k^{2}-1\right)\left[3(k+1) M_{k+1}-3(k-1) M_{k-1}+2 M_{k}\right]\right\}+\mathrm{O}\left(\delta^{2}\right), \\
\bar{\mu} s_{\alpha}^{3}(\hat{\mathbf{n}} \cdot \mathbf{V})(\alpha)= & \frac{1}{2}\left(4-M_{0}+3 M_{1}\right) T_{\circ}+\delta \cos (k \alpha)\left\{T _ { \circ } \left[2\left(k^{2}-1\right)-\frac{1}{4}\left(2 k^{2}-3\right) M_{k}-\frac{3}{4}\left(M_{1}-M_{0}\right)\right.\right. \\
& \left.+\frac{1}{2 k} N_{k}+\frac{3}{8 k}\left[(k-1)\left(2 k^{2}+4 k+1\right) M_{k+1}-(k+1)\left(2 k^{2}-4 k+1\right) M_{k-1}\right]\right] \\
+ & T_{k}\left[2+\frac{3}{4}\left((k+1) M_{k+1}-(k-1) M_{k-1}\right)-\frac{1}{2} M_{k}\right] \\
+ & \left.2 k^{2}\left(k^{2}-1\right)+\frac{k}{4}\left(k^{2}-1\right)\left[3(k+1) M_{k+1}+3(k-1) M_{k-1}-2 k M_{k}\right]\right\}+\mathrm{O}\left(\delta^{2}\right),
\end{aligned}
$$

where

$$
N_{k}(y)=M_{0}(y)+2 \sum_{j=1}^{k-1} M_{j}(y)+M_{k}(y),
$$

which is convergent in the limit $k \rightarrow \infty$ for non-zero $y$.

The instantaneous growth rate $\sigma_{k}$ is given by

$$
\bar{\mu} s_{\alpha}^{2} \sigma_{k}=-C_{1}(y ; k) T_{\circ}-C_{2}(y ; k) T_{k}-C_{3}(y ; k) s_{\alpha}^{-2},
$$


where the coefficients $C_{j}(y), j=1,2,3$, are given by

$$
\begin{aligned}
C_{1}(y ; k)= & 2 k^{2}+\frac{1}{4}\left[3 M_{0}(y)-\frac{\left(k^{2}-3\right)\left(2 k^{2}+1\right) M_{k}(y)+3\left(3 k^{2}-1\right) M_{1}(y)}{k^{2}-1}\right] \\
& +\frac{3}{8}\left[\left(2 k^{2}+4 k+1\right) M_{k+1}(y)+\left(2 k^{2}-4 k+1\right) M_{k-1}(y)\right], \\
C_{2}(y ; k)= & \frac{2 k^{2}}{k^{2}-1}+\frac{3 k}{4\left(k^{2}-1\right)}\left[(k+1)^{2} M_{k+1}(y)-(k-1)^{2} M_{k-1}(y)\right], \\
C_{3}(y ; k)= & 2 k^{4}-\frac{1}{2} k^{2}\left(k^{2}-1\right) M_{k}(y)+\frac{3 k^{2}}{4}\left[(k+1)^{2} M_{k+1}(y)+(k-1)^{2} M_{k-1}(y)\right],
\end{aligned}
$$

where we use the functions $M_{k}$ as defined in Appendix E.

The leading-order tension coefficient is given by

$$
T_{\circ}=-\frac{\bar{\mu} s_{\alpha} s_{\alpha t}}{2-\frac{1}{2} M_{0}(y)+\frac{3}{2} M_{1}(y)},
$$

which as expected is negative and growing exponentially in time. Note that $M_{1}(y) \rightarrow M_{0}(y)$ as $y \rightarrow 0$ and $T_{\circ}$ above is consistent with that obtained for the local drag model in Eq. (21). The next-order tension coefficient is given by

$$
T_{k}=-\frac{C_{5}(y ; k) T_{\circ}+C_{6}(y ; k) s_{\alpha}^{-2}}{C_{4}(y ; k)},
$$

where the coefficients are given by

$$
\begin{aligned}
C_{4}(y ; k)= & 2+\frac{1}{2}\left(k^{2}-1\right) M_{k}(y)+\frac{3}{4}\left[(k+1)^{2} M_{k+1}(y)+(k-1)^{2} M_{k-1}(y)\right], \\
C_{5}(y ; k)= & 4\left(k^{2}-1\right)+\frac{3}{2}\left(M_{k}(y)-M_{1}(y)\right)-\frac{k^{2}-1}{2 k} N_{k}(y) \\
& +\frac{3\left(k^{2}-1\right)}{8 k}\left[\left(2 k^{2}+4 k+1\right) M_{k+1}(y)-\left(2 k^{2}-4 k+1\right) M_{k-1}(y)\right], \\
C_{6}(y ; k)= & -2 k^{2}\left(k^{2}-1\right)+\frac{3}{4} k\left(k^{2}-1\right)\left[(k+1)^{2} M_{k+1}(y)-(k-1)^{2} M_{k-1}(y)\right] .
\end{aligned}
$$

The growth rate in the high-wavenumber limit $k \gg 1$ becomes

$$
\bar{\mu} s_{\alpha}^{4} \sigma_{k} \sim-k^{4}\left\{2-\frac{1}{2} M_{k}(y)+\frac{3}{4} M_{k+1}(y)+\frac{3}{4} M_{k-1}(y)\right\},
$$

where the behavior is dominated by the fourth-order elastic regularization of term $C_{3}(y ; k)$. Using the three-term recurrence relation of Eq. (E.5), one can show that the right-hand side of the above expression is always negative. Furthermore, by fixing $y$ and allowing $k \rightarrow \infty$, the toroidal harmonics decay to zero exponentially.

\section{Appendix D. Numerical methods}

Here we present the details of our numerical scheme. The $T$ and $\theta-\alpha$ are discretized using $N$ points uniformly in $\alpha$, where $h=2 \pi / N$ is the discretization length. Since the filament is a closed loop and $\theta(\pi, t)=\theta(-\pi, t)+2 \pi$, 
the linear term is subtracted off such that a straightforward application of DFT may be used. We rewrite the integral in Eq. (11) in terms of toroidal harmonics. In Appendix E, we present a fast and accurate method for numerically computing toroidal harmonics. Define the tensor

$$
\mathbf{B}\left(\alpha, \alpha+\alpha^{\prime}\right)=\mathbf{I}+\frac{1}{2}\left[\hat{\mathbf{s}}(\alpha) \hat{\mathbf{s}}\left(\alpha+\alpha^{\prime}\right)+\hat{\mathbf{s}}\left(\alpha+\alpha^{\prime}\right) \hat{\mathbf{s}}(\alpha)\right],
$$

and Eq. (11) becomes

$$
\begin{aligned}
\mathbf{K}[\mathbf{f}]= & \int_{-\pi}^{\pi} \frac{\mathbf{B}\left(\alpha, \alpha+\alpha^{\prime}\right) \cdot \mathbf{f}\left(\alpha+\alpha^{\prime}\right)-\mathbf{A}(\alpha) \cdot \mathbf{f}(\alpha)}{\left(4 \sin ^{2}\left(\alpha^{\prime} / 2\right)+y^{2}\right)^{1 / 2}} \mathrm{~d} \alpha^{\prime} \\
& +\int_{-\pi}^{\pi}\left[\frac{2 s_{\alpha}\left|\sin \left(\alpha^{\prime} / 2\right)\right|}{|\mathbf{R}|}(\mathbf{I}+\hat{\mathbf{R}} \hat{\mathbf{R}})-\mathbf{B}\left(\alpha, \alpha+\alpha^{\prime}\right)\right] \cdot \frac{\mathbf{f}\left(\alpha+\alpha^{\prime}\right) \mathrm{d} \alpha^{\prime}}{\left(4 \sin ^{2}\left(\alpha^{\prime} / 2\right)+y^{2}\right)^{1 / 2}} .
\end{aligned}
$$

The first integral is a convolution in $\alpha$ and is computed using a toroidal harmonic series in DFT space. The second integral is periodic, and is computed with spectral accuracy using the trapezoidal rule.

Preconditioning is used to improve the convergence rate when solving for $T$ using GMRES. One preconditioner we use is easily inverted using DFT,

$$
\mathcal{M}_{1} T=\left\{2 M_{0}(y) \partial_{\alpha}^{2}-\left(2+M_{0}(y)\right) \overline{\theta_{\alpha}^{2}}\right\} T,
$$

where $\overline{\theta_{\alpha}^{2}}=\int_{-\pi}^{\pi} \theta_{\alpha}^{2}(\alpha) \mathrm{d} \alpha /(2 \pi)$ is the mean of the squared curvature. Another preconditioner we use is applied in spectral space, and is the operator

$$
\tilde{\mathcal{M}}_{2} T_{k}=\left\{-2 M_{k}(y) k^{2}-\left(2+M_{k}(y)\right) \overline{\theta_{\alpha}^{2}}\right\} T_{k},
$$

where $T_{k}$ represents the $k$ th DFT mode of $T$. In our experience, the first preconditioner works well in the early stages of the simulation when the curve $\Gamma$ is far from self-approach. The second preconditioner works poorly in the earlier stages, but is superior to the first preconditioner on self-approach.

To illustrate the small-scale decomposition [17], we rewrite Eq. (18),

$$
\bar{\mu} \mathrm{e}^{4 t} \theta_{t}+\left(M_{0}(y)+2\right) \theta_{\alpha \alpha \alpha \alpha}=\mathbf{P}(\theta, T),
$$

and use DFT to obtain

$$
\left(v_{k}\left(t, t_{\circ}\right) \tilde{\theta}_{k}\right)_{t}=\frac{1}{\bar{\mu}} v_{k}\left(t, t_{\circ}\right) \mathrm{e}^{-4 t} \tilde{\mathbf{P}}_{k},
$$

where $\tilde{\theta}_{k}$ is the $k$ th DFT mode of $\theta-\alpha, \tilde{\mathbf{P}}_{k}$ represents the DFT of $\mathbf{P}(\theta, T)$, and $v_{k}$ represents the integrating factor

$$
v_{k}\left(t, t_{\circ}\right)=\exp \left(\int_{t_{\circ}}^{t} \frac{1}{\bar{\mu}}\left(M_{0}(y)+2\right) \mathrm{e}^{-4 t} k^{4} \mathrm{~d} t\right) .
$$

By numerically integrating Eq. (D.4), the linear small-scale behavior of the system is handled exactly in DFT space. Thus, the numerical manifestation of the fourth-order term now reflects its physical manifestation: it is stabilizing.

Let variables at time steps $t_{m}=m \Delta t$ for fixed $\Delta t$ be denoted by superscript $m$. With Adams-Bashforth method, we have

$$
\tilde{\theta}_{k}^{m+1}=v_{k}\left(t_{m}, t_{m+1}\right)\left\{\tilde{\theta}_{k}^{m}+\frac{\Delta t}{2 \bar{\mu}}\left[3 \mathrm{e}^{-4 t_{m}} \tilde{\mathbf{P}}^{m}-\mathrm{e}^{-4 t_{m-1}} v_{k}\left(t_{m-1}, t_{m}\right) \tilde{\mathbf{P}}^{m-1}\right]\right\},
$$

where we use the trapezoidal rule for the integral

$$
\int_{t_{m+1}}^{t_{m}} M_{0}(y) \mathrm{e}^{-4 t} \mathrm{~d} t=\frac{1}{2}\left(M_{0}\left(y_{m}\right) \mathrm{e}^{-4 t_{m}}+M_{0}\left(y_{m+1}\right) \mathrm{e}^{-4 t_{m+1}}\right)
$$


and where $y_{m}=\sqrt{\mathrm{e}} a \exp \left(-t_{m+1}\right)$. There exists an exact formula for the indefinite integral $\int^{t} M_{0}(y) \mathrm{e}^{-4 t}$ in terms of the first three toroidal harmonics $M_{0}(y), M_{1}(y)$ and $M_{2}(y)$. Using this formula for small $y$ and $\Delta t$ results in poor numerical accuracy due to round-off errors.

We find that the main restriction to the duration of our numerical runs comes from the eventual inaccuracies in calculating the second integral of Eq. (D.1). Essentially, the resolution may become inadequate in resolving $1 / R_{\circ}$ when the curve nears self-approach (see [1] for an analysis of a related problem). This under-resolution of the non-local term can lead to self-intersection of the filament or non-convergence in the GMRES.

To increase the resolution near peaks of $1 /|\mathbf{R}|$, we use interpolated values for $R_{\circ}$ and $\mathbf{f}\left(\alpha+\alpha^{\prime}\right)$. These interpolations need not be computed everywhere, but only local to those points along the curve nearing self-approach. Given the mesh points $\left\{\alpha_{j}\right\}_{j=1}^{N}$ along $\Gamma$, two points are considered sufficiently near self-approach if $R_{\circ}\left(\alpha_{j}, \alpha_{k}\right)<n_{I} \Delta s$ and $\bmod \left(\alpha_{j}-\alpha_{k}, 2 \pi\right)>n_{I} \Delta s$, where $n_{I}$ is specified as a run parameter. The second inequality prohibits the selection of nearly adjacent points along $\Gamma$. The part of the integral within the interval $\alpha^{\prime} \in\left[\alpha_{k}-\frac{1}{2} h, \alpha_{k}+\frac{1}{2} h\right]$ is then selected for interpolation. We interpolate $N_{I}$ points uniformly about $\alpha_{k}$ within this interval, where these points plus $\alpha_{k}$ are given by

$$
\alpha_{k, m}^{\prime}=\alpha_{k}+h\left(\frac{m}{N_{I}+2}-\frac{1}{2}\right)
$$

for $m=1,2, \ldots, N_{I}+1$. The integral is then approximated as

$$
\int_{\alpha_{k}-\frac{1}{2} h}^{\alpha_{k}+\frac{1}{2} h} F\left(\alpha_{j}, \alpha^{\prime}\right) \mathrm{d} \alpha^{\prime} \approx \frac{h}{N_{I}+1} \sum_{\alpha_{k, m}^{\prime}} F\left(\alpha, \alpha_{k, m}^{\prime}\right),
$$

where the trapezoidal rule is used.

Cubic splines are used to determine the interpolation, which results in the method becoming second-order in space (a DFT interpolation routine was also implemented, which would retain the spectral accuracy, but the cubic spline was chosen for efficiency reasons). In the comparison given in Fig. 3, the interpolation used $n_{I}=6$ and $N_{I}=12$. We use $N=4096$ for the run without interpolation, and half that number for the run with interpolation. Another simulation, not shown in Fig. 3, was run with no interpolation and $N=2048$. This led to insufficient resolution of the non-local term, and the errors led to a self-intersection of the filament at approximately $t=1.2$.

\section{Appendix E. Toroidal harmonics}

Local expansions along a weakly bent filament leads naturally to the use of "toroidal harmonics", or Legendre functions with zero order and integral+half degrees. They are used throughout Appendices A-E, in both the numerical scheme and linearized stability analysis. For notational convenience, we define the function

$$
M_{k}(y)=\int_{-\pi}^{\pi} \frac{\cos \left(k \alpha^{\prime}\right)}{\left(4 \sin ^{2}\left(\alpha^{\prime} / 2\right)+y^{2}\right)^{1 / 2}} \mathrm{~d} \alpha^{\prime} \quad \text { for } k=0,1,2, \ldots,
$$

where relation to Legendre functions (in notation corresponding to that used by Gradshtein and Ryzhik [14]) is given by $M_{k}(y)=2 Q_{k-1 / 2}^{0}\left(1+\frac{1}{2} y^{2}\right)$. With fixed $k$, the integral diverges logarithmically for small $y$. With fixed $y$, the $M_{k}$ decays to zero exponentially for large $k$.

A relatively fast and accurate method for computing these functions is required here. Since $y$ in the functions is parameterized by time in our model, the toroidal harmonic functions must be re-evaluated at each time step. 
Fettis [8] uses

$$
M_{k}\left(y_{j}\right)=\frac{\left(\begin{array}{c}
2 k \\
k
\end{array}\right) M_{0}\left(y_{j+1}\right)+2 \sum_{m=1}^{k}\left(\begin{array}{c}
2 k \\
k-m
\end{array}\right) M_{m}\left(y_{j+1}\right)}{\left(y_{j}+\left(y_{j}^{2}+4\right)^{1 / 2}\right)^{2 k-1}},
$$

where the quartic transformation

$$
y_{j+1}^{2}=y_{j}\left(y_{j}^{2}+4\right)^{1 / 2}\left(y_{j}+\left(y_{j}^{2}+4\right)^{1 / 2}\right)^{2}
$$

are used to obtain the toroidal harmonics from $1 \ll y$ asymptotic formula

$$
M_{k}(y) \sim \frac{\sqrt{\pi} \Gamma\left(k+\frac{1}{2}\right)}{\Gamma(k+1)}\left(\frac{2}{y+\left(y^{2}+4\right)^{1 / 2}}\right)^{2 k+1}+\mathrm{O}\left(\frac{1}{y^{4}}\right)
$$

for $k=0,1, \ldots$

Thiele gives a three-term recurrence relation for the set $\left\{M_{k}(y) ; k=0,1, \ldots\right\}$,

$$
(2 k-1) M_{k-1}(y)=2 k\left(y^{2}+2\right) M_{k}(y)-(2 k+1) M_{k+1}(y) .
$$

Using the recurrence upwards in $k$ is ill-conditioned, but downwards towards smaller $k$ is well-conditioned.

We combine both the methods. We begin with the large $y$ asymptotic formula, and compute the two largest $k$ modes using Fettis. The lower $k$ modes for that value of $y$ are then computed using Thiele recurrence. The quartic transformation and Fettis is then used to obtain two largest $k$ modes for a smaller $y$. We repeat the process as necessary.

Care must be taken when computing the largest $k$ modes, since underflow conditions on a computer are quickly approached for larger $y$. This, however, may be put to advantage, since not all $k$ modes necessarily need to be computed for the larger $y$, reducing the amount of computations required.

\section{References}

[1] G.R. Baker, M.S. Shelley, Boundary integral techniques for multi-connected domains, J. Comput. Phys. 64 (1986) 112.

[2] L. Bourdieu, T. Duke, M. Elowitz, D. Winkelmann, S. Leibler, A. Libchaber, Spiral defects in motility assays: a measure of motor protein force, Phys. Rev. Lett. 75 (1995) 176.

[3] P.N. Brown, A.C. Hindmarsh, Reduced storage matrix methods in stiff ODE systems, LLNL Report, UCRL-95088, 1987.

[4] S. Childress, Mechanics of Swimming and Flying, Cambridge University Press, Cambridge, 1981.

[5] A.T. Chwang, T.Y. Wu, Hydromechanics of low-Reynolds-number flow, Part 2. Singularity method for Stokes flows, J. Fluid Mech. 67 (1975) 787.

[6] P.G. de Gennes, J. Prost, The Physics of Liquid Crystals, Oxford University Press, Oxford, 1993.

[7] W. E, P. Palffy-Muhoray, Dynamics of filaments during the isotropic-smectic A phase transition, J. Nonlinear Sci. 9 (1999) $417-437$.

[8] H.E. Fettis, A new method for computing toroidal harmonics, Math. Comput. 24 (1970) 667-670.

[9] M. Gage, R. Hamilton, The heat equation shrinking convex plane curves, J. Diff. Geom. 23 (1986) 69.

[10] R. Goldstein, S. Langer, Nonlinear dynamics of stiff polymers, Phys. Rev. Lett. 75 (1995) 1094.

[11] R.E. Goldstein, T.R. Powers, C.H. Wiggins, Viscous nonlinear dynamics of twist and writhe, Phys. Rev. Lett. 80 (1998) $5232-5235$.

[12] A. Goriely, M. Tabor, Nonlinear dynamics of filaments I. Dynamical instabilities, Physica D 105 (1997) $20-44$.

[13] A. Goriely, M. Tabor, Nonlinear dynamics of filaments II. Nonlinear analysis, Physica D 105 (1997) 45-61.

[14] I.S. Gradshtein, I.M. Ryzhik, Table of Integrals, Series and Products, Academic Press, Boston, 1994.

[15] M. Grayson, The heat equation shrinks embedded plane curves to round points, J. Diff. Geom. 26 (1987) 285.

[16] G.J. Hancock, The self-propulsion of microscopic organisms through liquids, Proc. R. Soc. London, Ser. A 217 (1953) 96-121.

[17] T. Hou, J. Lowengrub, M.J. Shelley, Removing the stiffness from interfacial flows with surface tension, J. Comput. Phys. 114 (1994) 312-338.

[18] R. Johnson, An improved slender-body theory for Stokes flow, J. Fluid Mech. 99 (1980) 411.

[19] J. Keller, S. Rubinow, Slender-body theory for slow viscous flow, J. Fluid Mech. 75 (1976) $705-714$. 
[20] M.J. Lighthill, Mathematical Biofluid Dynamics, SIAM, Philadelphia, PA, 1975.

[21] N.H. Mendelson, J.J. Thwaites, J.O. Kessle, C. Li, Mechanics of bacterial macrofiber initiation, J. Bacteriol. 177 (1995) $7060-7069$.

[22] H. Naito, M. Okuda, O.-Y. Zhong-can, Pattern formation and instability of smectic-A filaments grown from an isotropic phase, Phys. Rev. E 55 (1997) 1655-1659.

[23] P. Palffy-Muhoray, B. Bergersen, H. Lin, R. Meyer, Z. Racz, Filaments in liquid crystals: structure and dynamics, in: S. Kai (Ed.), Pattern Formation in Complex Dissipative Systems, World Scientific, Singapore, 1991.

[24] R. Rogallo, NASA TM-73203, unpublished.

[25] A.S. Rudolph, B.R. Ratna, B. Kahn, Self-assembling phospholipid filaments, Nature 352 (1991) 52-55.

[26] Y. Saad, M. Schultz, GMRES: a generalized minimal residual algorithm for solving nonsymmetric linear systems, SIAM J. Sci. Statist. Comput. 7 (1986) 856.

[27] D. Schwendeman, A new numerical method for shock wave propagation based on geometrical shock dynamics, Proc. R. Soc. London A 441 (1993) 331-341.

[28] L.A. Segel, Mathematics Applied to Continuum Mechanics, Macmillan, New York, 1977.

[29] M.J. Shelley, T. Ueda, The nonlocal dynamics of stretching, buckling filaments, in: D.T. Papageorgiou, Y.Y. Renardy, A.V. Coward, S.-M. Sun (Eds.), Advances in Multi-Fluid Flows, SIAM, Philadelphia, PA, 1996, pp. 415-425.

[30] G.B. Whitham, A new approach to problems of shock dynamics, Part I: Two-dimensional problems, J. Fluid Mech. 2 (1957) 145. 